\title{
Systematic pan-cancer landscape identifies CARM1 as a potential prognostic and immunological biomarker
}

\author{
Yingqi Qiu ${ }^{1 \dagger}$, Hao Wang ${ }^{1 \dagger}$, Peiyun Liao ${ }^{1}$, Binyan $\mathrm{Xu}^{1}$, Rong Hu${ }^{1}$, Yulu Yang ${ }^{1}$ and Yuhua $\mathrm{Li}^{1} 2^{*}$
}

\begin{abstract}
Background: Belonging to the protein arginine methyltransferase (PRMT) family, the enzyme encoded by coactivator associated arginine methyltransferase 1 (CARM1) catalyzes the methylation of protein arginine residues, especially acts on histones and other chromatin related proteins, which is essential in regulating gene expression. Beyond its well-established involvement in the regulation of transcription, recent studies have revealed a novel role of CARM1 in tumorigenesis and development, but there is still a lack of systematic understanding of CARM1 in human cancers. An integrated analysis of CARM1 in pan-cancer may contribute to further explore its prognostic value and potential immunological function in tumor therapy.

Results: Based on systematic analysis of data in multiple databases, we firstly verified that CARM1 is highly expressed in most tumors compared with corresponding normal tissues, and is bound up with poor prognosis in some tumors. Subsequently, relevance between CARM1 expression level and tumor immune microenvironment is analyzed from the perspectives of tumor mutation burden, microsatellite instability, mismatch repair genes, methyltransferases genes, immune checkpoint genes and immune cells infiltration, indicating a potential relationship between CARM1 expression and tumor microenvironment. A gene enrichment analysis followed shortly, which implied that the role of CARM1 in tumor pathogenesis may be related to transcriptional imbalance and viral carcinogenesis.

Conclusions: Our first comprehensive bioinformatics analysis provides a broad molecular perspective on the role of CARM1 in various tumors, highlights its value in clinical prognosis and potential association with tumor immune microenvironment, which may furnish an immune based antitumor strategy to provide a reference for more accurate and personalized immunotherapy in the future.
\end{abstract}

Keywords: CARM1, Pan-cancer analysis, Prognosis, Immune, Tumor microenvironment

\section{Background}

Due to the heterogeneity and diversity of tumors, the deficiency of effective biomarkers represents one of the main bottlenecks restricting the development of cancer

\footnotetext{
*Correspondence: liyuhua1974@outlook.com

${ }^{\dagger}$ YYingqi Qiu and Hao Wang contributed equally to this work.

${ }^{2}$ Bioland Laboratory (Guangzhou Regenerative Medicine and Health

Guangdong Laboratory), Guangzhou, Guangdong 510005, People's

Republic of China

Full list of author information is available at the end of the article
}

medicine. The accumulated big data analysis of any gene of interest has become a powerful means to explore the complex process of tumorigenesis and development. Analyzing gene expression and related genetic modification allows us to evaluate its clinical prognosis and explore related signaling pathways, which could help to find new immunotherapy targets.

CARM1, also known as PRMT4, located in Chr19p13.2 (Fig. 1a), was first identified as an arginine methyltransferase that introduces asymmetric methylation of arginine residues in histone $\mathrm{H} 3$ and other

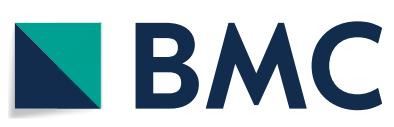

(c) The Author(s) 2022. Open Access This article is licensed under a Creative Commons Attribution 4.0 International License, which permits use, sharing, adaptation, distribution and reproduction in any medium or format, as long as you give appropriate credit to the original author(s) and the source, provide a link to the Creative Commons licence, and indicate if changes were made. The images or other third party material in this article are included in the article's Creative Commons licence, unless indicated otherwise in a credit line to the material. If material is not included in the article's Creative Commons licence and your intended use is not permitted by statutory regulation or exceeds the permitted use, you will need to obtain permission directly from the copyright holder. To view a copy of this licence, visit http://creativecommons.org/licenses/by/4.0/. The Creative Commons Public Domain Dedication waiver (http://creativeco mmons.org/publicdomain/zero/1.0/) applies to the data made available in this article, unless otherwise stated in a credit line to the data. 


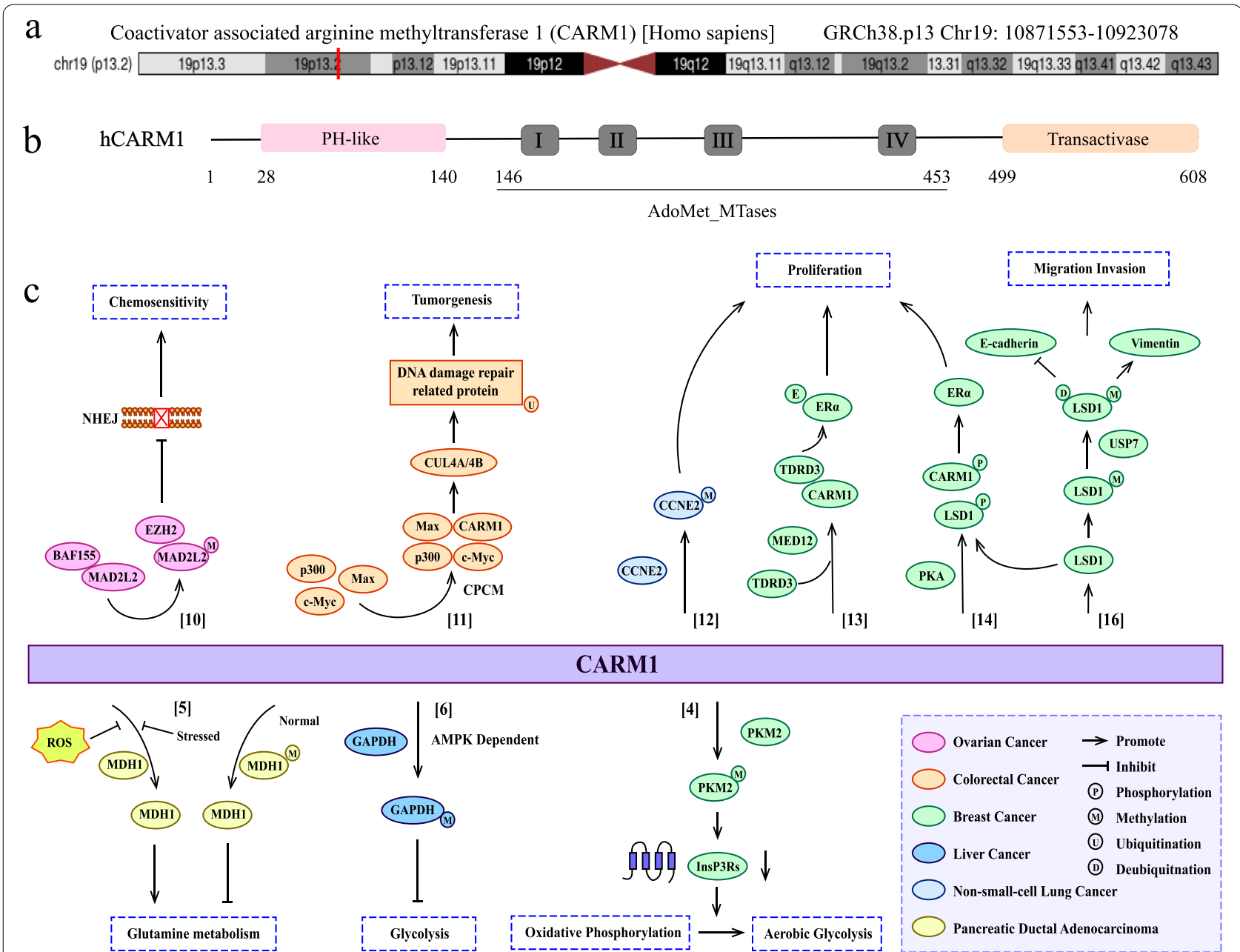

Fig. 1 Basic information about CARM1. a Genomic location of human CARM1. b Protein structure diagram of human CARM1. c According to the reported studies, the carcinogenic pathways CARM1 was involved in across different cancers are shown graphically. The related references are also indicated

chromatin-associated proteins [1]. With regard to human CARM1 protein, it is composed of an N-terminal pleckstrin homology-like domain (PH-like), a C-terminal transactivase domain, and a central catalytic domain containing the four conserved PRMT motifs (Fig. 1b). The $\mathrm{N}$ - and C-terminal domains of CARM1 are vital for substrate recognition and transcription-mediated activation [2], and the motifs in central catalytic domain are essential for binding of the cofactor S-adenosyl methionine (SAM) and the substrate arginine [3]. Long known as a transcriptional coactivator, recent studies have shown that it is also involved in the regulation of metabolism [4-6], autophagy [7], RNA regulation [8] and early mammalian development [9]. Recently, accumulating evidence has suggested that CARM1 also has an impact on the occurrence and development of tumors [10-14].
Existing studies on exploring the mechanisms of CARM1 methylation affecting tumor progression have shown that CARM1 is a coactivator of several cancer-related transcription factors and can be involved in promoting tumor cell proliferation and metastasis by methylating cancerrelated transcription factors, including NF- $\mathrm{kB}, \mathrm{p} 53$, steroid receptors and so on, and its high expression is associated with poor prognosis of tumors [15]. For example, in the most studied breast cancer, CARM1 could methylate the R838 site of lysine demethylase 1 (LSD1) to promote the binding of deubiquitinase USP7, resulting in the ubiquitination and stabilization of LSD1, thereby promoting the invasion and metastasis of breast cancer cells [16]. In addition, CARM1 has been found to be involved in regulating metabolic pathways in tumors. Metabolic reprogramming is a hallmark of cancer. In breast cancer 
cells, methylation of the key glycolytic enzyme pyruvate kinase M2 isoform (PKM2) by CARM1 shifts the metabolic balance from oxidative phosphorylation to aerobic glycolysis, producing a large amount of ATP, so as to promote tumor cell proliferation and migration [4]. Nevertheless, CARM1 is up-regulated when glucose starvation, followed by methylation of GAPDH to inhibiting glycolysis, thereby suppressing tumor cell proliferation in liver cancer cells [6], which is to some extent consistent with the results of the correlation analysis between CARM1 expression and liver cancer prognosis described in our work. Current evidence about effects of CARM1 on various cancers has been shown in Fig. $1 \mathrm{c}$ and Table 4 [5, 6, 13, 16-25].

However, researches on CARM1 in cancers are only started in recent years, and limited to several kinds of tumors. There is still no systematic pan-cancer evidence about the relationship between CARM1 and multiple tumor types based on big clinical data. Our work, for the first time, used multiple databases containing The Cancer Genome Atlas (TCGA) project, cBioPortal, Human Protein Altas (HPA) and so on to conduct a comprehensive pan-cancer analysis of CARM1. A group of factors, such as gene expression, survival status, genetic alteration, tumor mutation burden (TMB), microsatellite instability (MSI), methyltransferases genes, immune infiltration, and relevant cellular pathway, are included to investigate the potential associations between CARM1 and the pathogenesis and clinical prognosis of different cancers, providing a basis for further understanding the role of CARM1 in tumor immunotherapy.

\section{Results}

\section{Expression profile of CARM1 across normal tissues and cancer samples}

In this work, we aimed to investigate the role of human CARM1 in tumorigenesis and development. As mentioned above, CARM1 protein is usually composed of a $\mathrm{N}$-terminal $\mathrm{pH}$ like domain (cl17171), a C-terminal transactivase domain, and a central catalytic domain (cl17173), and its structure is conserved among most species (Fig. S1a, see Additional file 1, e.g., H. sapiens, $M$. mulatta, $R$. norvegicus, etc.). The evolutionary relationship of CARM1 protein among different species is also shown in the phylogenetic tree data (Fig. S1b).

Firstly, the physiologic CARM1 gene expression levels across normal tissues were observed combining HPA, GTEx and Function annotation of the mammalian genome 5 (FANTOM5) datasets. As shown in Fig. 2a, CARM1 expression is the highest in skeletal muscle with high RNA tissue specificity, while other detected tissues express relatively low level of CARM1, especially the blood cell lineage. When analyzing the expression of CARM1 in different blood cells, a low RNA blood cell specificity could be observed (Fig. S2b, see Additional file 2). The CARM1 expression levels in various cancer cell lines were also analyzed. The result shows that all cancers expressed CARM1, with the highest expression level in ovarian cancer, followed by endometrial cancer and colorectal cancer (Fig. S2a).

Next, the TIMER2 approach was applied to compare the expression difference of CARM1 between various cancer types and corresponding normal tissues. Among them, primary cancers show significantly higher expression levels than normal tissues in bladder urothelial carcinoma (BLCA), breast invasive carcinoma (BRCA), cholangio carcinoma (CHOL), colon adenocarcinoma (COAD), esophageal carcinoma (ESCA), head and neck squamous cell carcinoma (HNSC), liver hepatocellular carcinoma (LIHC), lung adenocarcinoma (LUAD), lung squamous cell carcinoma (LUSC), rectum adenocarcinoma (READ), stomach adenocarcinoma (STAD), thyroid carcinoma (THCA), uterine corpus endometrial carcinoma (UCEC) $(P<0.001)$, and pheochromocytoma and paraganglioma (PCPG), prostate adenocarcinoma (PRAD) $(P<0.01)$. In contrast, CARM1 is downregulated in tumor relative to normal tissues in kidney Chromophobe $(\mathrm{KICH})$ and kidney renal clear cell carcinoma (KIRC) $(P<0.001)$ (Fig. 2b). After combining the normal tissue of the GTEx dataset as controls, the diffuse large B-cell lymphoma (DLBC) and thymoma (THYM) cohorts show upregulated expression levels, while there is no significant difference between the remaining tumor types and the corresponding normal tissues (Fig. 2c).

In addition, HPA, TCGA and CPTAC datasets were used to evaluate CARM1 expression at protein level. We obtained the immunohistochemistry (IHC) results from HPA and compared them with the CARM1 gene expression data provided by TCGA. As shown in Fig. 3a, the analysis results from the two databases are basically consistent. The staining results of BRCA, LUAD, LUSC present strong or medium CARM1 staining, while the corresponding normal tissues show low or moderate staining. On the contrary, normal kidney tissues have low or moderate staining, while $\mathrm{KICH}$ samples have no CARM1 staining. Furthermore, the results of the CPTAC dataset indicate higher expression of CARM1 protein in the primary tissues of KIRC and colon cancer than in normal tissues (Fig. 3b), and increase from grade I to grade II in KIRC patients (Fig. S2c). It is noteworthy that although there are no significant correlations of protein expression between primary tissues of breast cancer, ovarian cancer, lung adenocarcinoma, UCEC and related normal tissues, the expression of protein in normal and other subtypes of breast cancer is significantly higher than that of luminal subtype. In addition, the CARM1 protein expression 


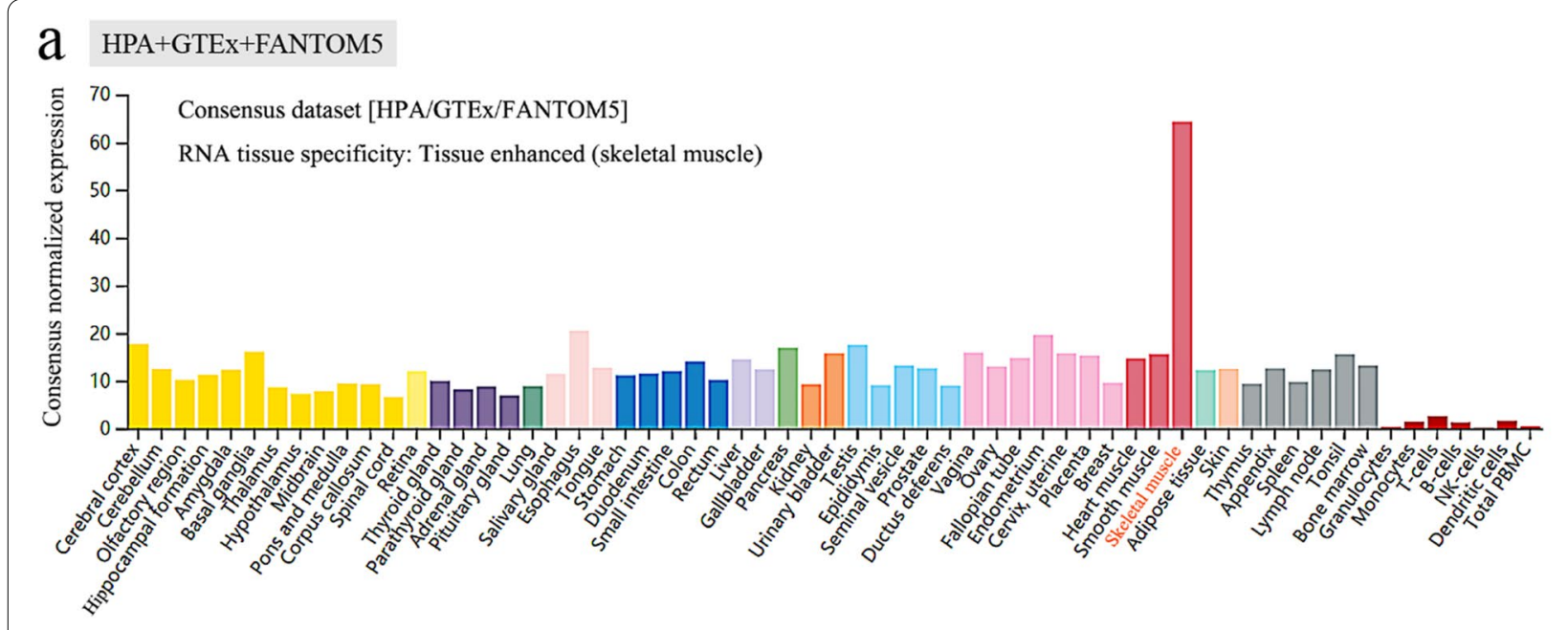

b TCGA Dataset

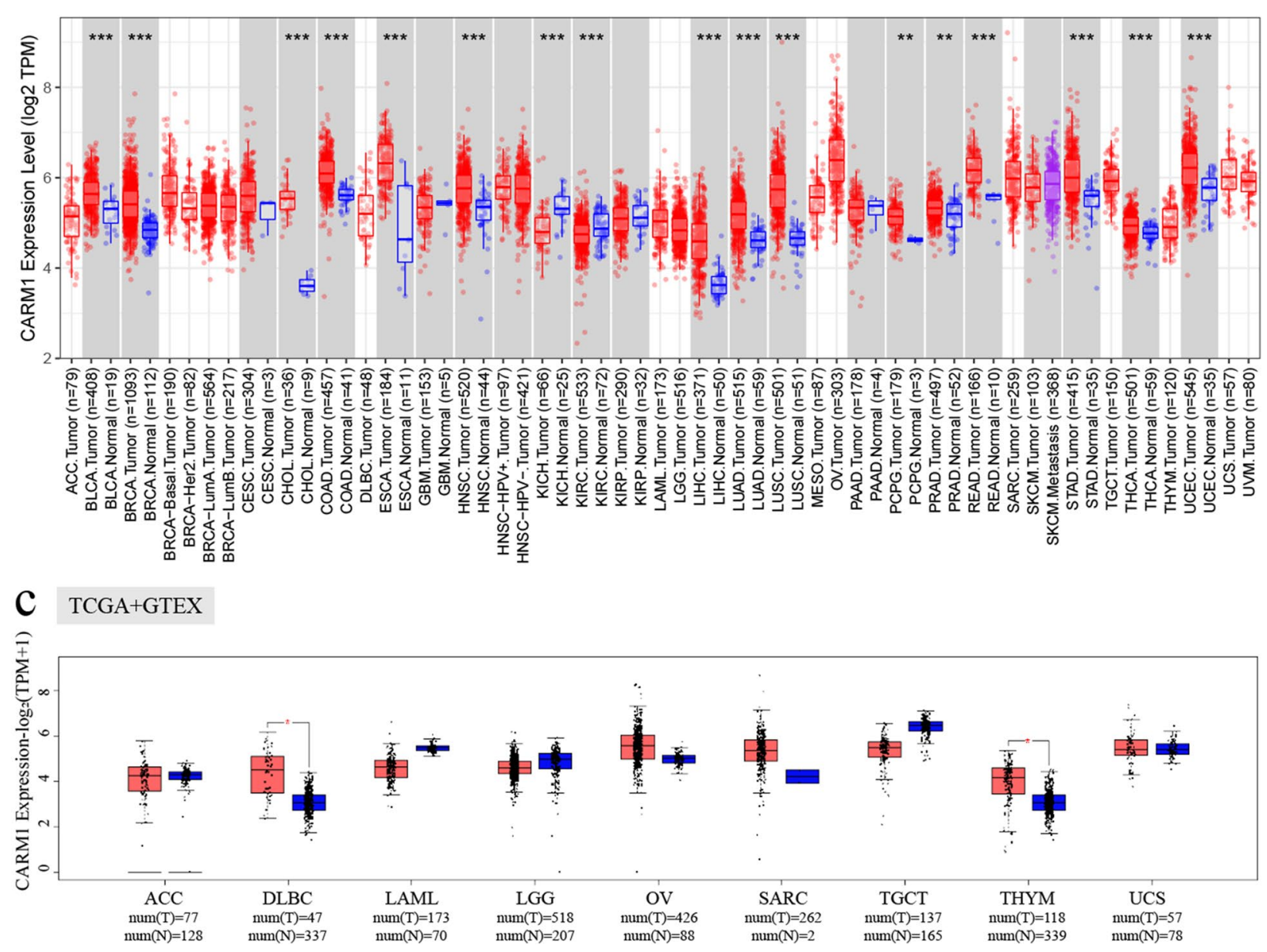

Fig. 2 Expression profile of CARM1. a CARM1 expression in normal tissues. $\mathbf{b}$ The expression of CARM1 in tumors and normal tissues from TCGA project were compared by TIMER2. ${ }^{*} P<0.05$, **P $<0.01$, ${ }^{* *} P<0.001$. c For the types of ACC, DLBC, LGG, OV, SARC, TGCT, THYM and UCS in TCGA project, the corresponding normal tissues in GTEx database were used as controls. ${ }^{*} P<0.05$ 


\section{a}
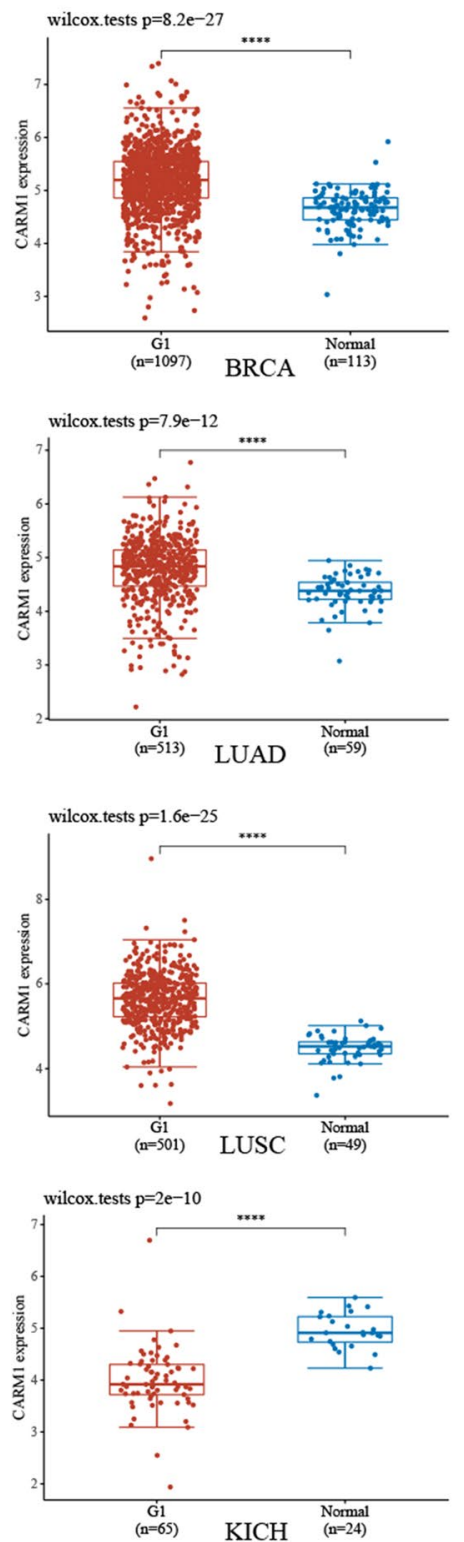
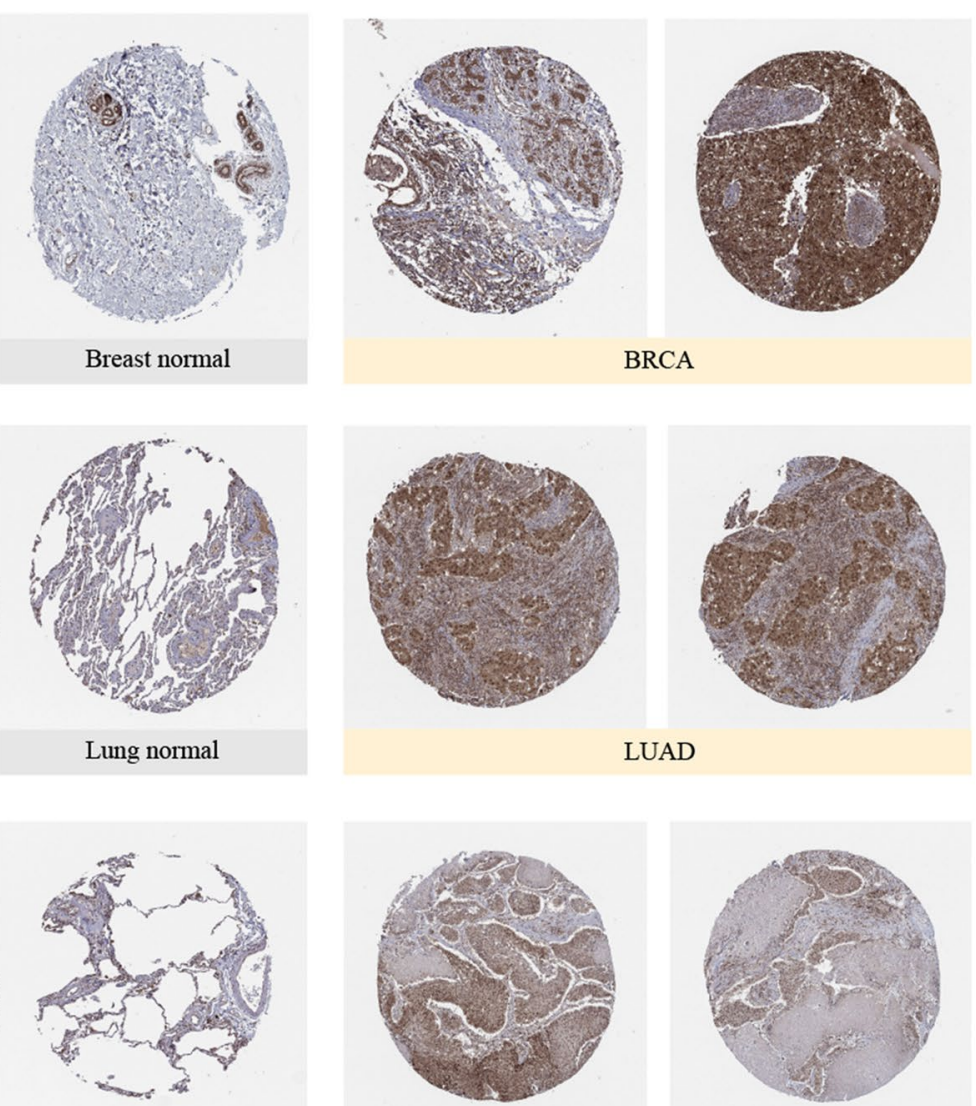

Lung normal

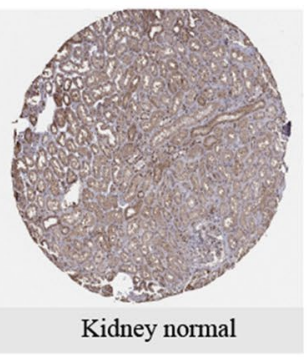

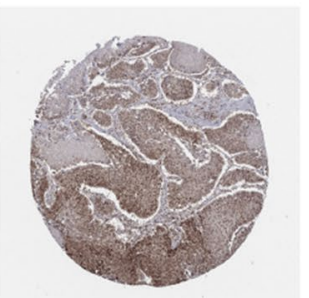

LUSC
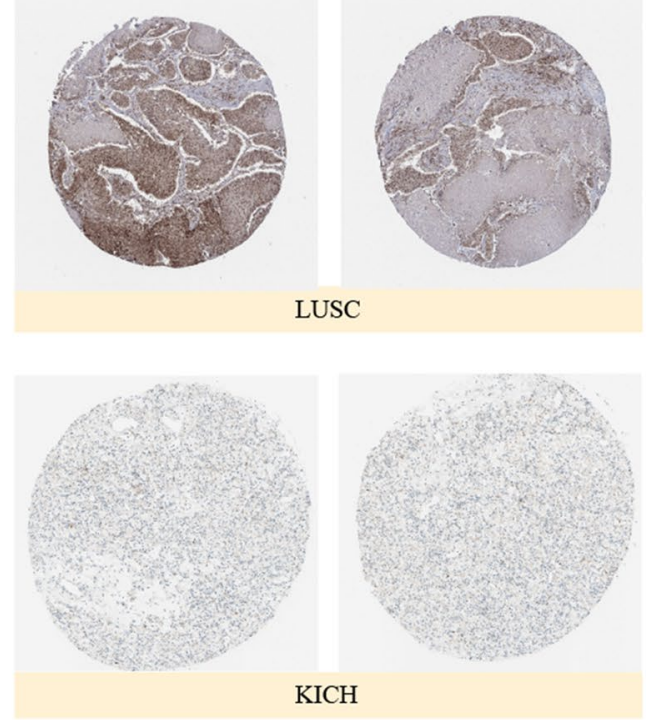

$\mathrm{KICH}$ b

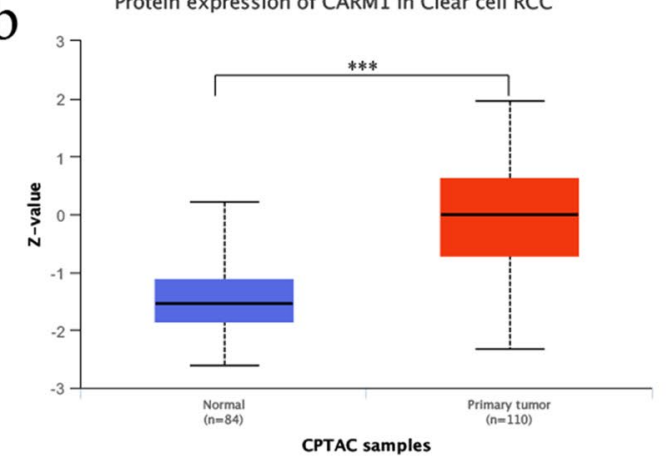

Protein expression of CARM1 in Colon cancer

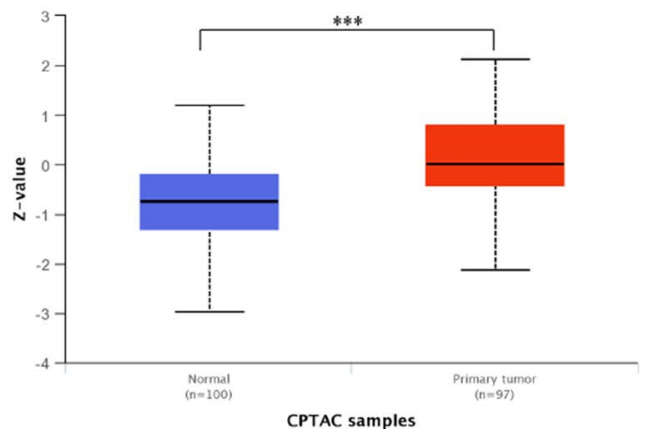

Fig. 3 Analysis of CARM1 total protein expression data. a Comparison of CARM1 gene expression data from TCGA (left) with IHC results of HPA (right). The CARM1 RNA expression is up-regulated in BRCA, LUAD, LUSC and down-regulated in $\mathrm{KICH}$, which is consistent with the results of IHC. $\mathbf{b}$ Data from CPTAC dataset indicate KIRC and colon cancer samples express higher level of CARM1 total protein than normal tissues. ${ }^{* * * P}<0.001$ 

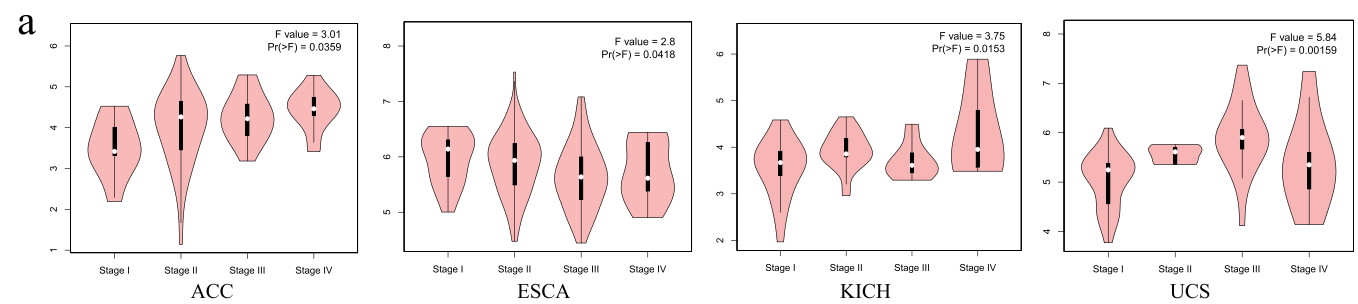

b

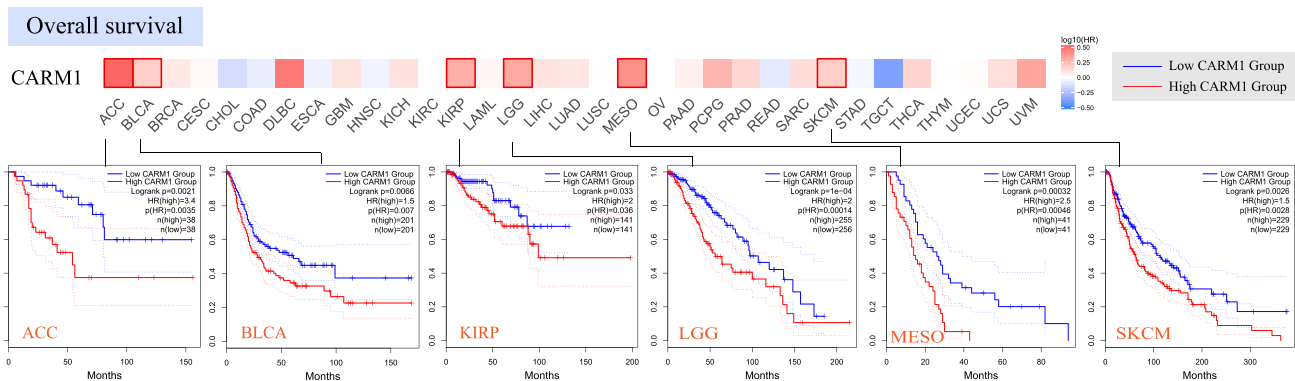

C Desease free survival

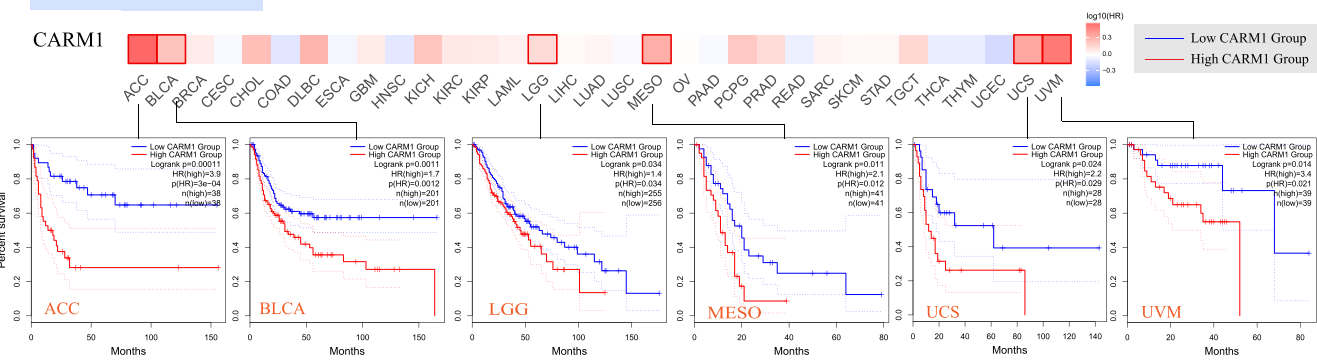

Fig. 4 Correlation analysis of CARM1 gene expression with different pathological stages and survival prognosis across cancers in TCGA. a Based on data from TCGA, CARM1 gene expression levels were analyzed according to the main pathological stages. Log $_{2}($ TPM +1 ) was applied for log-scale. GEPIA2 tool was used to perform overall survival (b) and disease-free survival (c) analysis of cancers in TCGA according to CARM1 gene expression. The survival map and Kaplan-Meier curve of positive results are given

level of 21-40years old group in ovarian cancer patients is up-regulated compared with other age groups, which may be a potential feature of this group.

Using the "Pathological Stage Plot" module of GEPIA2, we also found that the expression of CARM1 is related to the pathological stages of the following carcinomas, comprising ACC (adrenocortical carcinoma), ESCA, KICH and UCS (uterine carcinosarcoma) (Fig. 4a, all $P<0.05$ ), but no significant difference is observed in other tumors (Fig. S3, see Additional file 3).

\section{Prognostic value of CARM1 in pan-cancers}

To explore the correlation between CARM1 expression and prognosis of patients with different tumors, TCGA and GEO were used and cancer cases were divided into high-expression and low-expression groups according to the expression levels of CARM1. As shown in Fig. 4b, highly expressed CARM1 is linked to poor prognosis of overall survival (OS) for cancers such as
ACC $(P=0.0021)$, BLCA $(P=0.0066)$, KIRP (kidney renal papillary cell carcinoma, $P=0.033$ ), LGG (Brain lower Grade Glioma, $P=0.00014$ ), MESO (Mesothelioma, $P=0.00046$ ), SKCM (skin cutaneous melanoma, $P=0.0026$ ). Analysis results of DFS presented a correlation between high CARM1 expression and poor prognosis of ACC $(P=0.00011)$, BLCA $(P=0.0012)$, LGG $(P=0.034)$, MESO $(P=0.012)$, UCS $(P=0.029)$ and UVM (uveal melanoma, $P=0.021$ ) (Fig. 4c).

Furthermore, Kaplan-Meier Plotter tool was also used to identify the prognostic value of CARM1 in the five types of tumors shown in Fig. S4 (see Additional file 8), which manifest a correlation between high expression CARM1 and poor OS, PPS, FP prognosis for gastric cancer and lung cancer. As for ovarian cancer, low CARM1 expression is related to poor PFS, while the relationship between CARM1 expression and OS, PPS prognosis are not detected. Additionally, CARM1 is a high-risk gene in breast cancer (OS, $P=0.019$; DMFS, $P=0.00033$; PPS, $P=0.0038$ ), while it is a low-risk 
Table 1 Subgroup analysis on the correlation of CARM1 expression and prognosis of breast cancer cases

\begin{tabular}{|c|c|c|c|c|c|c|c|c|c|c|}
\hline \multirow[t]{2}{*}{ Factor } & \multirow[t]{2}{*}{ Subgroup } & \multirow[t]{2}{*}{ Sample size } & \multicolumn{2}{|l|}{ os } & \multicolumn{2}{|l|}{ RFS } & \multicolumn{2}{|c|}{ DMFS } & \multicolumn{2}{|l|}{ PPS } \\
\hline & & & HR & $P$ & HR & $P$ & HR & $P$ & HR & $P$ \\
\hline \multirow[t]{2}{*}{ ER status-IHC } & ER positive & 3499 & 1.45 & 0.028 & 1.25 & 0.0068 & 1.26 & 0.26 & 1.84 & 0.0023 \\
\hline & ER negative & 2168 & 1.28 & 0.17 & 1.2 & 0.095 & 1.53 & 0.0021 & 1.3 & 0.3 \\
\hline \multirow[t]{2}{*}{ ER status-array } & ER positive & 5526 & 1.32 & 0.062 & 1.15 & 0.042 & 1.33 & 0.0036 & 1.52 & 0.013 \\
\hline & ER negative & 2009 & 1.27 & 0.13 & 0.77 & 0.013 & 1.22 & 0.14 & 1.83 & 0.012 \\
\hline \multirow[t]{2}{*}{ TP53 status } & mutated & 272 & 0.71 & 0.33 & 0.64 & 0.066 & 0.38 & 0.02 & 5.55 & 0.0015 \\
\hline & Wild type & 388 & 0.65 & 0.17 & 1.68 & 0.02 & 0.47 & 0.035 & 2.08 & 0.048 \\
\hline \multirow[t]{2}{*}{ PR status } & PR positive & 1559 & 1.52 & 0.27 & 1.61 & 0.0025 & 1.46 & 0.11 & 4.98 & 0.085 \\
\hline & PR negative & 1989 & 0.54 & 0.042 & 1.14 & 0.25 & 1.37 & 0.038 & 0.32 & 0.022 \\
\hline \multirow[t]{2}{*}{ HER2 status } & HER2 positive & 1273 & 1.29 & 0.18 & 0.79 & 0.047 & 1.77 & 0.00077 & 1.27 & 0.31 \\
\hline & HER2 negative & 6262 & 1.28 & 0.03 & 1.11 & 0.092 & 1.26 & 0.01 & 1.48 & 0.0039 \\
\hline \multirow[t]{3}{*}{ Grade } & Grade 1 & 576 & 0.24 & 0.041 & 1.59 & 0.18 & 4.89 & 0.018 & 0.5 & 0.23 \\
\hline & Grade 2 & 1795 & 1.63 & 0.021 & 1.41 & 0.0037 & 1.38 & 0.031 & 1.97 & 0.0055 \\
\hline & Grade 3 & 2058 & 1.34 & 0.073 & 1.18 & 0.085 & 1.26 & 0.084 & 1.75 & 0.007 \\
\hline \multirow[t]{4}{*}{ Intrinsic subtype } & Basal & 1494 & 1.49 & 0.038 & 0.79 & 0.068 & 1.25 & 0.16 & 2.01 & 0.022 \\
\hline & Luminal A & 3511 & 1.32 & 0.16 & 0.88 & 0.12 & 1.31 & 0.038 & 1.77 & 0.014 \\
\hline & Luminal B & 2015 & 1.36 & 0.083 & 1.16 & 0.11 & 1.5 & 0.0071 & 1.56 & 0.12 \\
\hline & HER2+ & 515 & 0.71 & 0.25 & 0.62 & 0.0079 & 1.54 & 0.086 & 1.81 & 0.12 \\
\hline \multirow[t]{2}{*}{ Lymph node status } & Lymph node positive & 2153 & 1.2 & 0.3 & 0.86 & 0.12 & 0.83 & 0.19 & 1.77 & 0.0055 \\
\hline & Lymph node negative & 2829 & 1.71 & 0.018 & 1.4 & 0.00011 & 1.51 & 0.001 & 1.66 & 0.017 \\
\hline \multirow[t]{6}{*}{ Pietenpol subtype } & Basal-like 1 & 418 & 2.02 & 0.1 & 0.53 & 0.014 & 0.7 & 0.26 & 2.39 & 0.2 \\
\hline & Basal-like 2 & 165 & 4.51 & 0.0012 & 0.68 & 0.22 & 1.49 & 0.3 & & \\
\hline & immunomodulatory & 462 & 0.66 & 0.3 & 0.71 & 0.21 & 2.02 & 0.05 & 0.27 & 0.035 \\
\hline & Mesenchymal & 382 & 0.57 & 0.17 & 0.58 & 0.03 & 1.72 & 0.073 & 3.75 & 0.033 \\
\hline & Mesenchymal stem-like & 201 & 0.32 & 0.024 & 0.54 & 0.11 & 1.76 & 0.28 & & \\
\hline & Luminal androgen receptor & 413 & 0.8 & 0.46 & 0.71 & 0.094 & 1.89 & 0.043 & 1.67 & 0.26 \\
\hline
\end{tabular}

$E R$ Estrogen receptor, $P R$ Progesterone receptor, HER2 human epidermal growth factor receptor 2, NA not available data, HR hazard ratio

The $P$ value marked bold indicates that the prognosis of the low expression group is better than that of the high expression group, while the $P$ value marked bold and italic indicates that the prognosis of the high expression group is better than that of the low expression group. NS, $P>0.05 ;{ }^{*} P<0.05 ;{ }^{* *} P<0.01 ;{ }^{* * *} P<0.001$

gene in liver cancer (DSS, $P=0.04$; RFS, $P=0.033$; PFS, $P=0.013)$.

We also performed a subgroup survival analysis using selected clinical factors and observed different conclusions. Significantly, highly expressed CARM1 is linked to poor prognosis for estrogen receptor (ER) positive subgroup of breast cancer cases. As for patients in grade II or lymph node negative status, CARM1 overexpression may be a poor prognostic factor (Table 1). For gastric cancer patients with lymph node metastasis, highly expressed CARM1 is associated with poor OS, FP and PPS prognosis (Table S1, see Additional file 4). Notably, consistent with the overall analysis results of liver cancer cases aforementioned, CARM1 overexpression is a beneficial prognostic factor in most subgroup analyses, especially in patients with hepatitis virus infection or in low grade, and it may turn into a deleterious prognostic factor when the disease developed into high grade (Table S2, see
Additional file 5). More details about prognosis of these five tumors can be found in Table 1, Table S1-S4 (see Additional files 4, 5, 6, 7).

\section{Genetic alteration analysis of CARM1 across cancers}

Gene alteration features of different cancers in TCGA were further investigated using the cBioPortal tool. Among all cancers, ovarian cancers present the highest alteration frequency of CARM1 $(>8 \%)$ with "amplification" as the primary type (Fig. 5a). It is worth noting that all cases of uterine carcinosarcoma $(\sim 7 \%)$, adrenocortical carcinoma $(\sim 3.5 \%)$ and mesothelioma $(>2 \%)$ with gene variation have "amplification" mutation type, while all cases of DLBCL ( $>4 \%$ ) have copy number deletion of CARM1. Figure 5b further shows the types, loci and number of cases of CARM1 gene variations. As the main type of gene change, there are 65 missenses in CARM1, among which 419 sites in the methyltransferase domain 
a

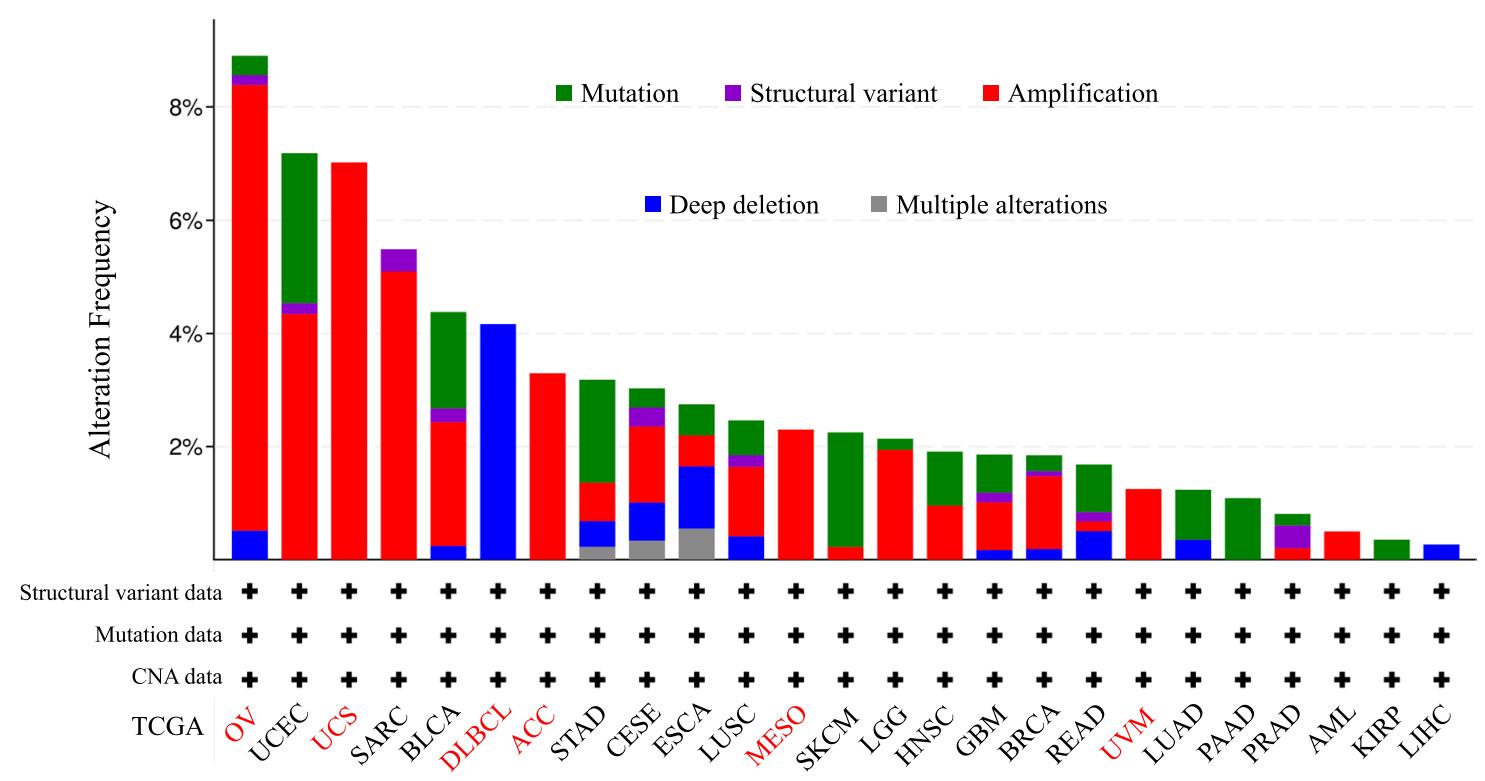

b

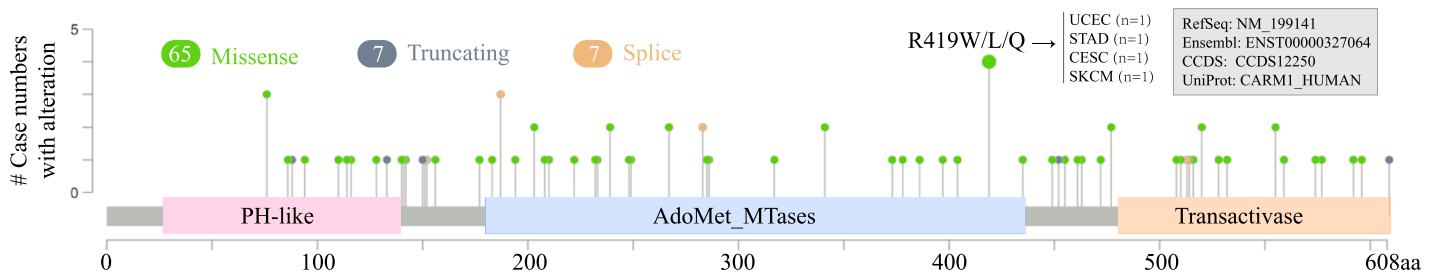

c
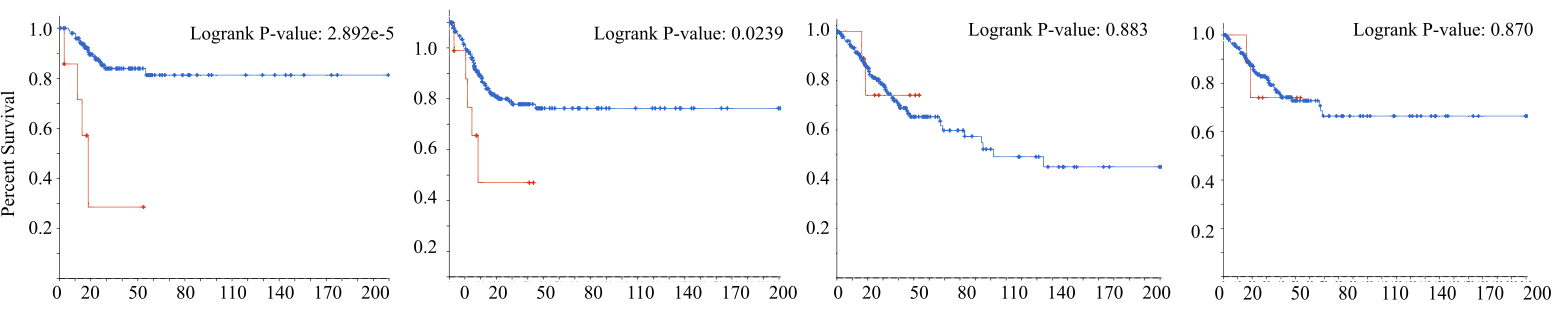

Fig. 5 Mutation features of CARM1 in different cancers of TCGA. The cBioPortal tool was used to analyze the mutation features of CARM1. a The alteration frequency of different mutation types of CARM1 gene in different cancers. $\mathbf{b}$ The mutation types, sites and case number of the CARM1 genetic alteration were further presented. c Potential correlation between mutation status and DFS, PFS, OS and DSS rate of CESC. DFS, disease-free survival; PFS, progress-free survival; OS, overall survival; DSS, disease-specific survival

had the maximum $\mathrm{R}$ (arginine) mutations, translation from R to W (tryptophan), L (leucine) and Q (glutamine), respectively. Moreover, the potential association between CARM1 genetic alterations and clinical prognosis was analyzed in various cancers cases (Table 2). Compared with the cases with CARM1 change, Cervical squamous cell carcinoma and endocervical adenocarcinoma (CESC) cases without altered CARM1 show better prognosis in PFS $(P=0.0239)$ and DFS $(P=2.892 e-5)$, but not OS $(P=0.883)$ and DSS $(P=0.870)$. These results have been shown in Fig. $5 \mathrm{c}$. In addition, the prognosis of the group without genetic changes is significantly better than that of the relative group in COAD.

Serving as emerging prognostic and immunotherapeutic response biomarkers for a variety of tumors, the quantification of TMB and MSI have entered the exploratory stage of clinical transformation [26, 27]. Herein, we analyzed the correlation between CARM1 mRNA expression and TMB, MSI. As shown in Fig. 6a, there is a positive correlation between CARM1 expression and TMB for LUAD $(P=0.00031)$, pancreatic adenocarcinoma (PAAD, $P=0.049$ ), SARC 
Table 2 Summary of CARM1 genetic alteration and clinical survival prognosis in various cancers cases

\begin{tabular}{llllll}
\hline Tumor types & Sample size & $\boldsymbol{P}$ & & & \\
\cline { 3 - 6 } & & OS & PFS & DSS & DFS \\
\hline BLCA & 562 & 0.743 & NA & NA & $\mathbf{0 . 0 4 6 5}$ \\
BRCA & 963 & 0.793 & NA & NA & 0.469 \\
CESC & 278 & 0.883 & $\mathbf{0 . 0 2 3 9}$ & 0.87 & $\mathbf{0 . 0 0 0 0 3}$ \\
COAD & 436 & 0.521 & $\mathbf{0 . 0 2 0 2}$ & 0.845 & $\mathbf{0 . 0 3 1 4}$ \\
SKCM & 363 & 0.077 & 0.191 & 0.145 & NA \\
GBM & 378 & 0.356 & 0.501 & 0.273 & NA \\
HNSC & 385 & $\mathbf{0 . 0 0 1 2}$ & NA & NA & NA \\
LUAD & 230 & 0.334 & NA & NA & 0.055 \\
LUSC & 469 & 0.62 & 0.44 & 0.263 & 0.583 \\
PRAD & 489 & 0.515 & 0.29 & 0.667 & $\mathbf{0 . 0 4 3 2}$ \\
STAD & 434 & $\mathbf{0 . 0 3 1 5}$ & 0.277 & 0.132 & 0.448 \\
UCS & 509 & 0.535 & 0.147 & 0.298 & 0.263
\end{tabular}

$P$ value derived from Log Rank test. The bold font indicates that the prognosis of the mutated group is worse than that of the non-mutated group. NS, $P>0.05$; * $P<0.05 ;{ }^{* *} P<0.01 ; * * * 0.001$

$(P=0.0067)$, BRCA $(P=0.0013), \quad$ STAD $\quad(P=8.3 e-$ 07), SKCM $(P=0.021)$, HNSC $(P=0.0059)$, LGG $(P=4.1 e-06)$ and ACC $(P=0.0038)$, but a negative correlation for $\operatorname{KIRP}(P=0.032)$, LIHC $(P=0.036)$, THCA $(P=0.00022)$. As for MSI, CARM1 expression is also positively correlated with LUAD $(P=0.022)$, LUSC $(P=0.00028)$, SARC (sarcoma, $P=0.0029)$,
STAD $(P=0.017)$ and UVM $(P=0.014)$, but is negatively correlated with that of SKCM $(\mathrm{P}=0.0038)$, HNSC $(P=0.0022)$, READ $(P=1.3 e-07)$ and DLBC $(P=0.0044)$. It should be noted that both TMB and MSI of LUAD, SARC and STAD are positively correlated with CARM1 expression, which deserves further study.

\section{CARM1 expression is related to DNA repair genes and methyltransferase expression in various tumor samples}

Correlation between mutation indexes TMB, MSI and CARM expression prompted us to further explore the potential relationship between CARM1 expression and tumorigenesis mechanism. MMRs, the intracellular mismatch repair mechanisms, the loss of function of its key genes will lead to unrepairable DNA replication errors, and then result in somatic mutations [28]. Here, utilizing TCGA expression profile data, we evaluated the relationship between CARM1 expression and mutation of five major MMRs genes including MLH1, MSH2, MSH6, PMS2, EPCAM. Except COAD, AML (acute myeloid leukemia), READ, UCS and UVM, the expression of CARM1 is positively correlated with MMRs genes mutation in almost all types of tumors from TCGA, and the results of MLH1, MSH2, MSH6 and PMS2 are more significant (Fig. 7a).

As a form of DNA chemical modification involving the transfer of methyl group onto the $\mathrm{C} 5$ position of the

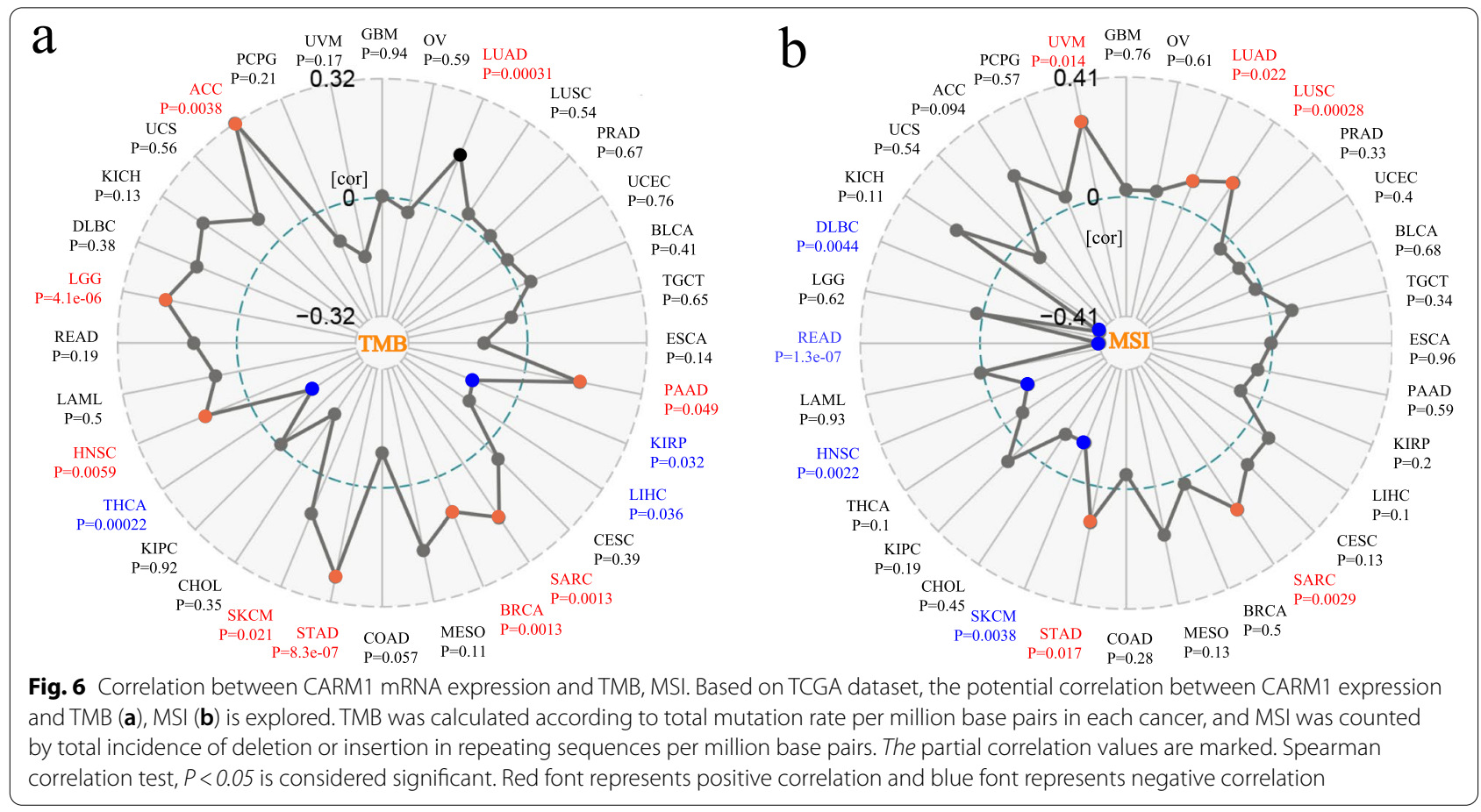




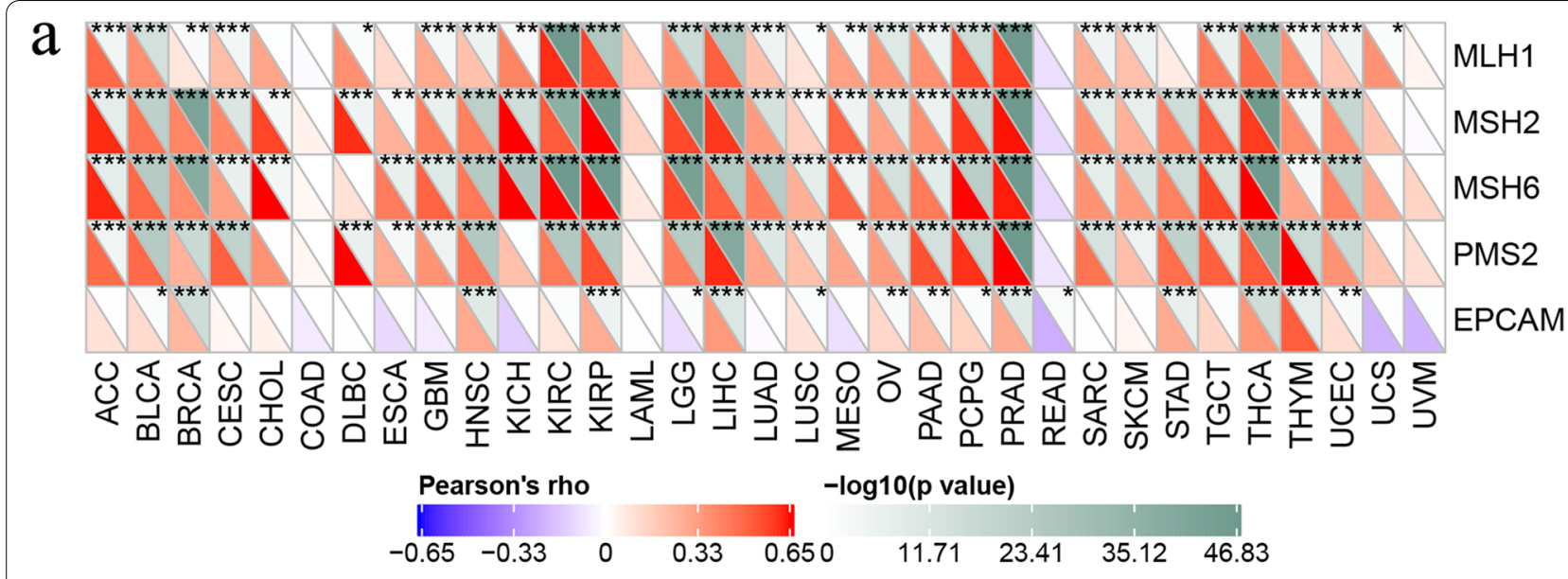

b

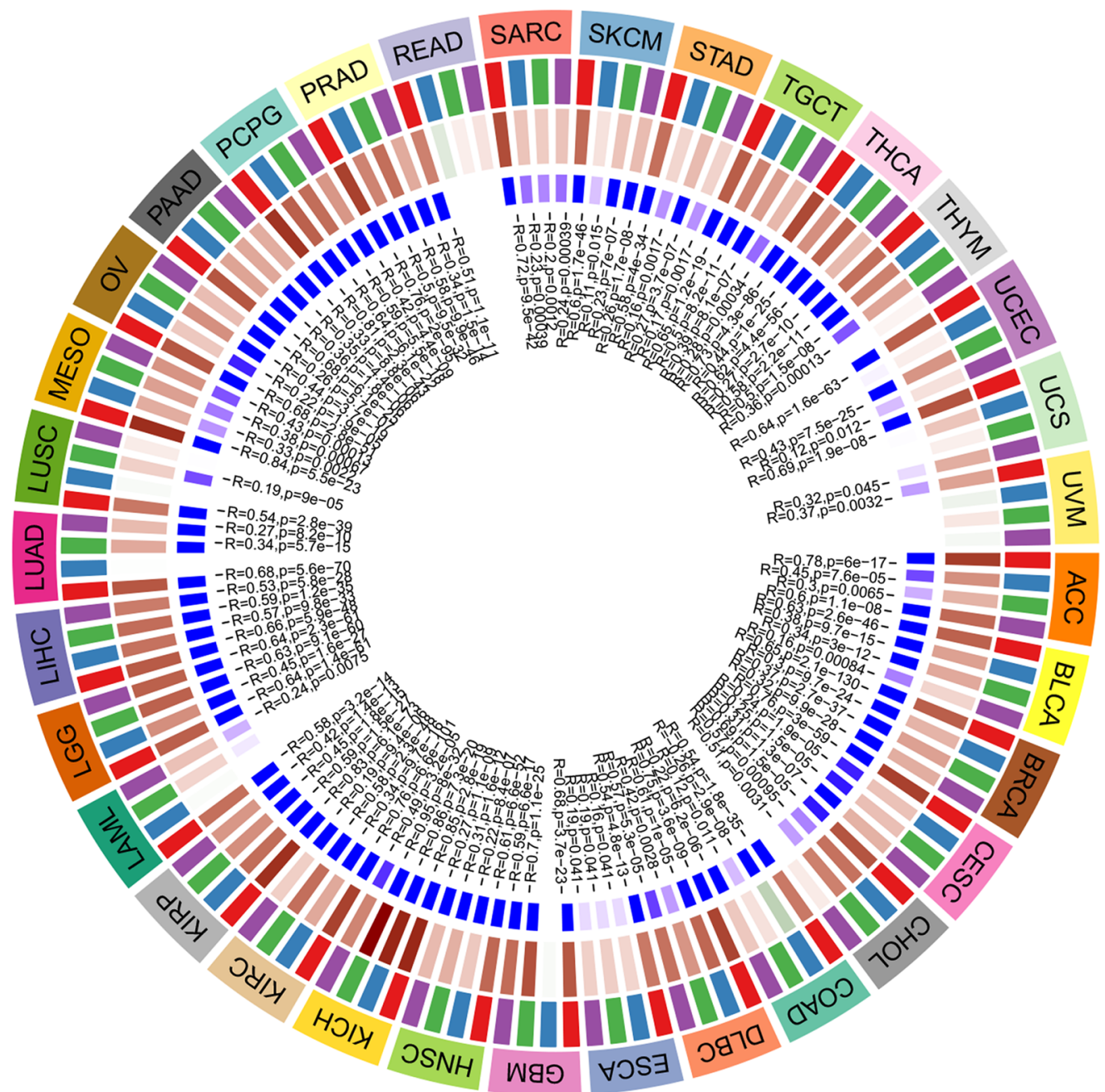

Fig. 7 Correlation analysis of CARM1 expression with MMR genes mutation and methyltransferases genes expression. a Correlation between CARM1 mRNA expression and five major MMR genes mutation. The lower triangle in each block represents the coefficient calculated by Pearson's correlation test, and the upper triangle represents $\log _{10}$ transformed $P$-value. ${ }^{*} P<0.05$, ${ }^{*} P<0.01$, ${ }^{* *} P<0.001$. b Co-expression analysis of CARM1 and methyltransferases. The outer circle represents different tumor types, the second circle represents the four methyltransferases (DNMT1: red, DNMT2: blue, Dnmt3a: green, DNMT3b: Purple), the third circle represents co-expression correlation coefficient, and the fourth circle represents $P$ value. $P<0.05$ is considered significant 
cytosine to form 5-methylcytosine under the action of DNA methyltransferase, DNA methylation can change genetic performance without changing DNA sequence, which can change chromatin structure, DNA conformation, DNA stability and the interaction between DNA and protein, so as to regulate gene expression [29]. Here, analysis of the correlation between CARM1 and four methyltransferases (DNMT1, DNMT2, DNMT3a, DNMT3b) expression was conducted for each tumor to explore whether CARM1 expression is related to epigenetics. The result shows that CARM1 and methyltransferases are significantly co-expressed in almost all tumors (Fig. 7b), which is an interesting phenomenon worth further exploring.

\section{Correlation between TME and CARM1 expression Correlation between tumor-infiltrating immune cells and CARM1 expression}

Tumor microenvironment (TME) is an environment conducive to tumor cell survival established by tumor cells that evade early immune surveillance by remodeling local immune cells and stromal cells, and a full understanding of TME provides us with valuable clues to develop more effective therapeutic strategies. Currently, there is still a lack of research on the association of CARM1 methylation and immune cell infiltration. In an impressive study, CARM1 inactivation was found to activate innate immunity in melanoma resistant cell lines with high CARM1 expression, making them more sensitive to $\mathrm{T}$ cell immunity and immune checkpoint blockade [30]. Moreover, it is eye-catching that CARM1-KO T cells exert more effective antitumor effects than wild-type, indicating that CARM1 inhibition enables immunotherapy of resistant tumors by dual effects on tumor cells and T cells, which has great clinical translational value. And the analysis results presented below in this study also manifest there is a large correlation between immune cells infiltration and CARM1 expression in the TME of some tumors, pointing out that investigating the role of CARM1 in the field of tumor immunity in-depth is a direction worth exploring.

A growing number of researches show that tumor-infiltrating immune cells serve as a vital part in TME, affecting the occurrence and development of tumors significantly $[31,32]$. It is of great significance to further explore the pan cancerous relationship between these immune cells and CARM1 expression. As a significant part of TME, cancer-associated fibroblasts in tumor stroma have been reported to be involved in regulating the function of a variety of tumor infiltrating immune cells [33]. Therefore, several algorithms were applied to investigate the relationship between CARM1 expression and cancer-associated fibroblasts infiltration. As shown in Fig. 8a, in most TCGA tumors, the expression of CARM1 is positively correlated with the infiltration of cancer-related fibroblasts, especially in ACC, KIRC, MESO, THYM and UVM. The representative scatterplots produced using one algorithm are also presented in the Figure.

Because tumor infiltrating lymphocytes are independent predictors of sentinel lymph node status and survival in cancer [32], analogous analysis on potential correlation between $\mathrm{CD}^{+} \mathrm{T}$ cells infiltration and CARM1 expression is also conducted. The results show that $\mathrm{CD} 8^{+} \mathrm{T}$ cells infiltration is negatively correlated with CARM1 expression in ESCA, HNSC, LUSC, PAAD, SKCM and THYM cases (Fig. 8b). Similar trend could be found between CARM1 expression and other immune cells infiltration in ESCA, LUSC, SKCM and THYM in Table 3, which presents more comprehensive details. On the contrary, a positive correlation trend could be observed in BLCA, BRCA, KIRC, KIRP, LGG, LIHC, LUAD, PAAD, PCPG, PRAD and THCA.

\section{Correlation between TME scores and CARM1 expression}

In order to conduct more in-depth research on the pancancer relationship between TME and CARM1 expression, ESTIMATE algorithm was used to analyze the relationship between stromal and immune scores and gene expression level among 33 tumors from TCGA. The results of the top three tumors with the highest correlation coefficient have been shown in Fig. 9, which reveal that CARM1 expression is significantly negatively correlated with immune scores in LUSC, SARC, testicular germ cell tumors (TGCT), indicating that the content of immune cells decreases while the level of CARM1 expression escalates (Fig. 9a). Similar results were also observed in the stromal score of LUSC and SARC, while the opposite correlation was shown in KIRC (Fig. 9b).

Genetic instability of tumor cells often leads to a large number of mutations, and the expression of nonsynonymous mutations could produce tumor specific antigens called tumor neoantigens [34]. Because they are not expressed in normal tissues, neoantigens have high immunogenicity and can activate $\mathrm{T}$ cells to trigger immune response, which have become a potential new target of tumor immunotherapy. Here, we counted the

(See figure on next page.)

Fig. 8 Different algorithms were used to explore the potential correlation between CARM1 expression and immune infiltration across all types of cancer in TCGA. a Correlation heat map and representative scatterplots of cancer-associated fibroblasts infiltration, indicating that CARM1 expression is positively correlated with the cancer-related fibroblasts infiltration in most tumors, especially in ACC, KIRC, MESO, THYM and UVM. b Correlation heat map and representative scatterplots of CD8+ T cell, showing that CD8+ T cells infiltration is negatively correlated with CARM1 expression in ESCA, HNSC, LUSC, PAAD, SKCM and THYM. $P<0.05$ is considered significant 
a

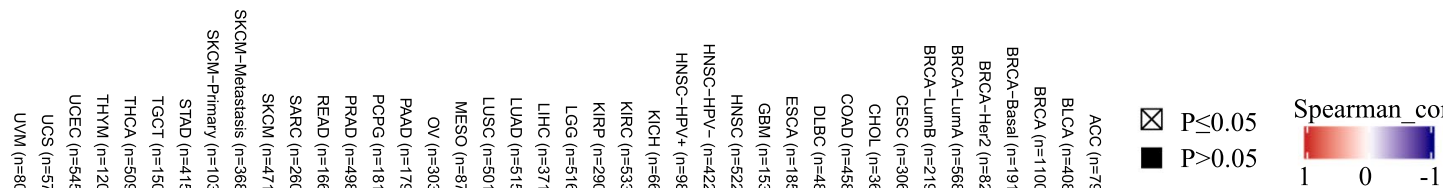

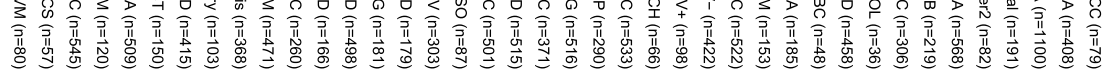

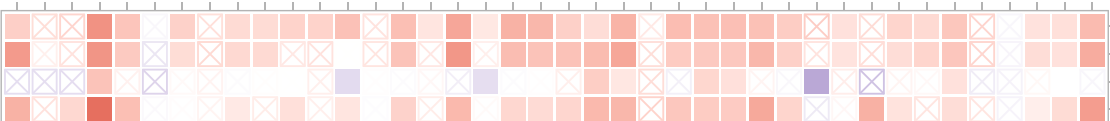

EPIC

MCPCOUNTER XCELL
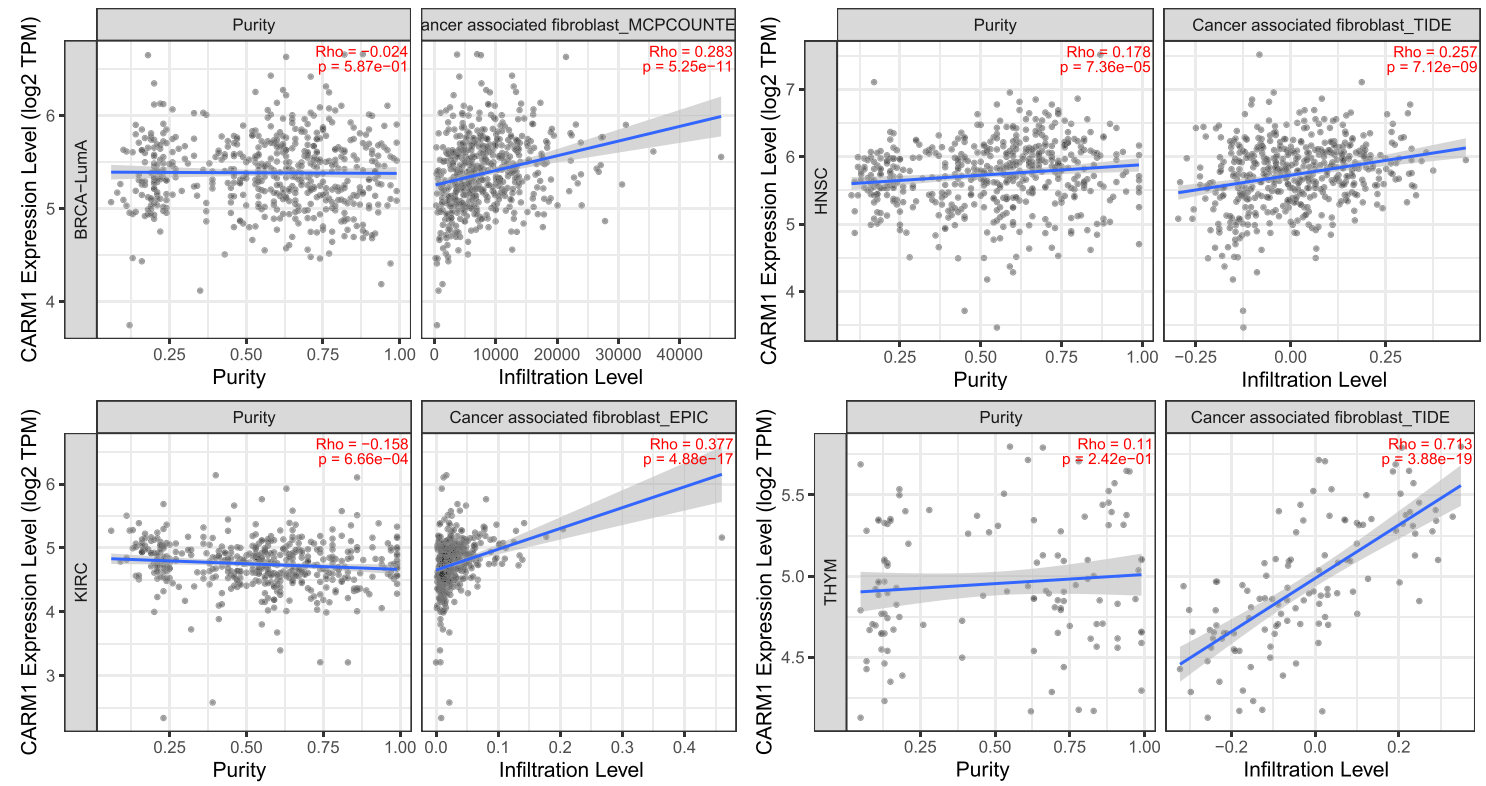

b
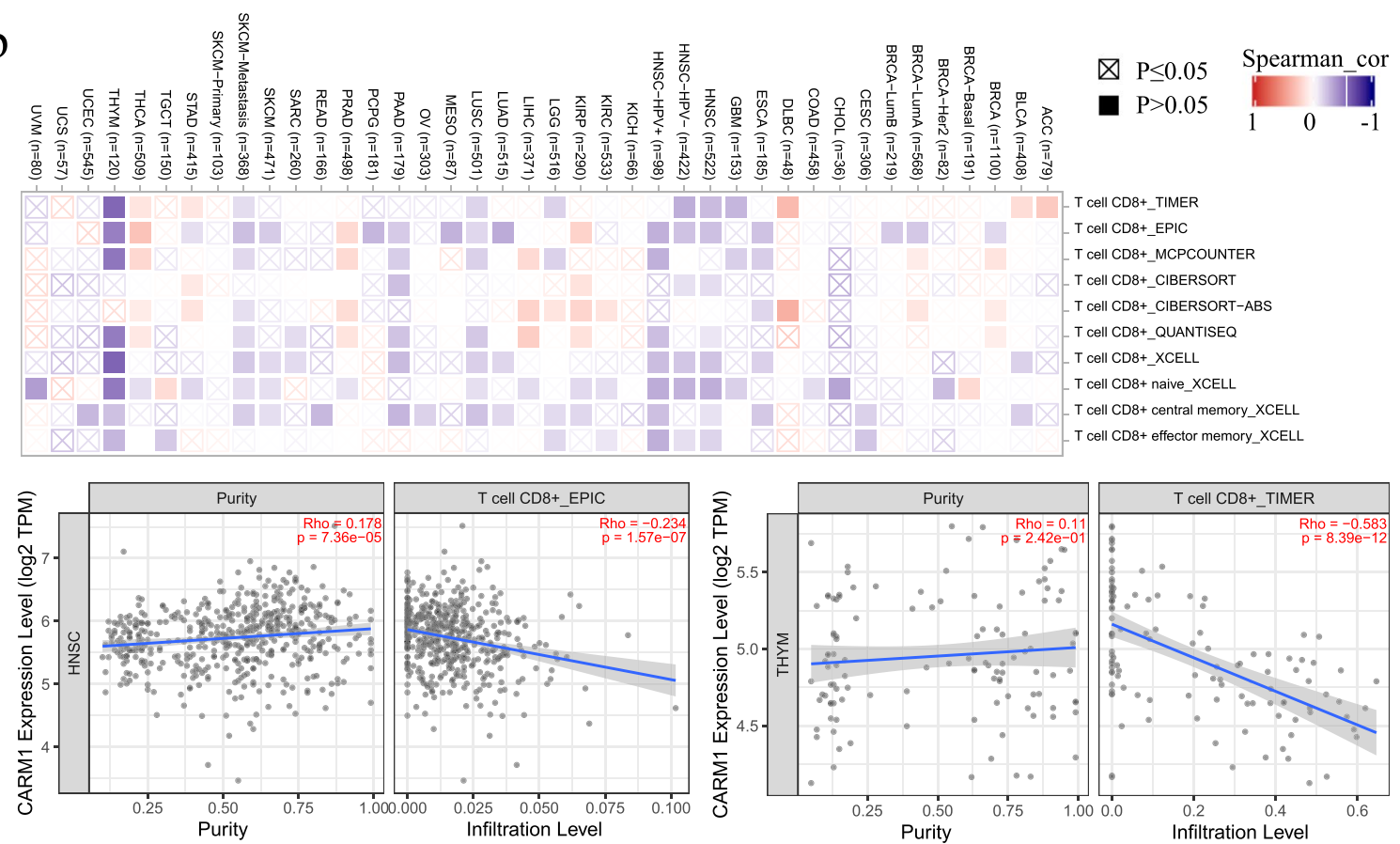

Fig. 8 (See legend on previous page.) 
Table 3 Correlation analysis between CARM1 expression and other immune cells infiltration

\begin{tabular}{|c|c|c|c|c|c|c|}
\hline Tumor type & B cell & CD4+ T cell & CD8 $+\mathrm{T}$ cell & Neutrophil & Macrophage & Dendritic \\
\hline ACC & $0.357 / * * *$ & $-0.006 / \mathrm{ns}$ & $-0.023 / \mathrm{ns}$ & $0.149 / 0.19$ & $0.047 / \mathrm{ns}$ & $0.243 / *$ \\
\hline BLCA & $-0.011 / \mathrm{ns}$ & $0.017 / \mathrm{ns}$ & $0.262 /^{* * *}$ & $0.152 /{ }^{* * *}$ & $0.086 / \mathrm{ns}$ & $0.209 / * * *$ \\
\hline BRCA & $0.104 / * * *$ & $0.19 / * * *$ & $0.096 / * *$ & $0.206 / * * *$ & $0.09 / * *$ & $0.168 / * * *$ \\
\hline CESE & $-0.065 / \mathrm{ns}$ & $0.069 / \mathrm{ns}$ & $-0.134 / *$ & $-0.083 / \mathrm{ns}$ & $-0.178 / * *$ & $-0.022 / \mathrm{ns}$ \\
\hline $\mathrm{CHOL}$ & $0.139 / \mathrm{ns}$ & $0.106 / \mathrm{ns}$ & $-0.15 / \mathrm{ns}$ & $0.459 / * *$ & $0.267 / \mathrm{ns}$ & $0.101 / \mathrm{ns}$ \\
\hline COAD & $-0.157 / * * *$ & $0.141 / * *$ & $-0.267 / * * *$ & $-0.012 / \mathrm{ns}$ & $-0.044 / \mathrm{ns}$ & $-0.003 / \mathrm{ns}$ \\
\hline DLBC & $0.089 / \mathrm{ns}$ & $0.096 / \mathrm{ns}$ & $-0.504 /^{* *}$ & $0.4 /^{*}$ & $0.192 / \mathrm{ns}$ & $-0.035 / \mathrm{ns}$ \\
\hline ESCA & $-0.102 / \mathrm{ns}$ & $-0.103 / \mathrm{ns}$ & $-0.295 / * * *$ & $-0.22 /^{* *}$ & $-0.174 / *$ & $-0.128 / \mathrm{ns}$ \\
\hline GBM & $-0.014 / \mathrm{ns}$ & $-0.11 / \mathrm{ns}$ & $-0.047 / \mathrm{ns}$ & $-0.043 / \mathrm{ns}$ & $-0.036 / \mathrm{ns}$ & $-0.091 / \mathrm{ns}$ \\
\hline HNSC & $-0.047 / \mathrm{ns}$ & $-0.198 / * * *$ & $-0.073 / \mathrm{ns}$ & $0.046 / \mathrm{ns}$ & $0.076 / \mathrm{ns}$ & $0.065 / \mathrm{ns}$ \\
\hline $\mathrm{KICH}$ & $0.082 / n s$ & $0.065 / \mathrm{ns}$ & $0.311 /^{*}$ & $-0.091 / \mathrm{ns}$ & $0.446 / * * *$ & $0.109 / \mathrm{ns}$ \\
\hline $\mathrm{KIRC}$ & $0.264 / * * *$ & $0.45 / * * *$ & $0.203 / * * *$ & $0.402 / * * *$ & $0.399 / * * *$ & $0.43 / * * *$ \\
\hline KIRP & $0.117 / *$ & $0.097 / \mathrm{ns}$ & $0.079 / \mathrm{ns}$ & $0.187 / * *$ & $0.01 / \mathrm{ns}$ & $0.226 / * * *$ \\
\hline LGG & $0.331 / * * *$ & $0.198 / * * *$ & $0.228 / * * *$ & $0.383 / * * *$ & $0.226 / * * *$ & $0.315 / * * *$ \\
\hline LIHC & $0.309 / * * *$ & $0.441 / \mathrm{ns}$ & $0.111 / *$ & $0.321 / * * *$ & $0.348 / * * *$ & $0.301 / * * *$ \\
\hline LUAD & $-0.049 / \mathrm{ns}$ & $0.235 / * * *$ & $-0.044 / \mathrm{ns}$ & $0.179 / * * *$ & $0.113 /^{*}$ & $0.151 / * * *$ \\
\hline LUSC & $-0.147 / * * *$ & $-0.024 / \mathrm{ns}$ & $-0.302 / * * *$ & $-0.292 / * * *$ & $-0.198 / * * *$ & $-0.249 / * * *$ \\
\hline MESO & $0.266 /^{*}$ & $0.073 / \mathrm{ns}$ & $0.108 / \mathrm{ns}$ & $-0.267 / *$ & $0.119 / \mathrm{ns}$ & $0.306 / * *$ \\
\hline OV & $0.035 / \mathrm{ns}$ & $-0.041 / \mathrm{ns}$ & $-0.04 / \mathrm{ns}$ & $-0.049 / \mathrm{ns}$ & $0.094 / \mathrm{ns}$ & $0.006 / \mathrm{ns}$ \\
\hline PAAD & $0.204 /^{* *}$ & $0.094 / \mathrm{ns}$ & $0.331 / * * *$ & $0.38 / * * *$ & $0.426 / * * *$ & $0.375 / * * *$ \\
\hline PCPG & $0.106 / \mathrm{ns}$ & $0.15 / *$ & $0.124 / \mathrm{ns}$ & $0.227 / * *$ & $0.313 / * * *$ & $0.179 / *$ \\
\hline PRAD & $0.405 / * * *$ & $0.111 / *$ & $0.112 / * * *$ & $0.313 / * * *$ & $0.352 / * * *$ & $00.33 / * * *$ \\
\hline READ & $-0.064 / \mathrm{ns}$ & $0.125 / \mathrm{ns}$ & $-0.346 / * * *$ & $-0.245 /^{* *}$ & $-0.162 /^{*}$ & $0.01 / \mathrm{ns}$ \\
\hline SARC & $-0.134 /^{*}$ & $-0.333 / * * *$ & $-0.139 / *$ & $-0.096 / \mathrm{ns}$ & $-0.217 / * * *$ & $-0.307 / /^{* *}$ \\
\hline SKCM & $-0.149 / * *$ & $-0.004 /^{*}$ & $-0.208 / * * *$ & $-0.125 /^{* * *}$ & $-0.036 / \mathrm{ns}$ & $0.144 / * *$ \\
\hline STAD & $-0.103 / *$ & $0.002 / \mathrm{ns}$ & $-0.116 / *$ & $-0.1 / \mathrm{ns}$ & $-0.123 /^{*}$ & $-0.072 / \mathrm{ns}$ \\
\hline TGCT & $0.119 / \mathrm{ns}$ & $-0.28 /^{* * *}$ & $0.137 / \mathrm{ns}$ & $-0.252 /^{* *}$ & $0.024 / \mathrm{ns}$ & $-0.009 / \mathrm{ns}$ \\
\hline THCA & $0.577 / * * *$ & $0.66 / * * *$ & $-0.343 /^{* * *}$ & $0.458 / * * *$ & $0.504 / * * *$ & $0.403 / * * *$ \\
\hline THYM & $-0.008 / \mathrm{ns}$ & $-0.437 / * * *$ & $-0.179 / \mathrm{ns}$ & $0.252 / * *$ & $0.005 / \mathrm{ns}$ & $-0.255 / * *$ \\
\hline UCEC & $-0.097 / *$ & $-0.076 / \mathrm{ns}$ & $-0.075 / \mathrm{ns}$ & $0.033 / \mathrm{ns}$ & $-0.148 / * * *$ & $-0.023 / \mathrm{ns}$ \\
\hline UCS & $-0.087 / \mathrm{ns}$ & $-0.186 / \mathrm{ns}$ & $0.048 / \mathrm{ns}$ & $-0.002 / \mathrm{ns}$ & $0.261 / \mathrm{ns}$ & $0.335 /^{*}$ \\
\hline UVM & $-0.093 / \mathrm{ns}$ & $0.034 / \mathrm{ns}$ & $0.12 / \mathrm{ns}$ & $-0.061 / \mathrm{ns}$ & $0.032 / \mathrm{ns}$ & $0.254 / *$ \\
\hline
\end{tabular}

NS $P>0.05, * P<0.05, * * P<0.01, * * * P<0.001$

number of neoantigens in each tumor sample, and analyzed the relationship between them and the expression of CARM1. As shown in Fig. 9c, there is a significant positive correlation between CARM1 expression and the number of immune neoantigens in BRCA, STAD and HNSC, suggesting a new idea of immunotherapy.

\section{Correlation between immune checkpoints and CARM1 expression}

Tumor cells induce immunosuppression through various ways to achieve immune escape, and tumor immunotherapy is a treatment method to control and eliminate tumors by restarting and maintaining tumor immune cycle and restoring normal anti-tumor immune response, in which immune checkpoint inhibitor is an important aspect [35]. Herein, we also conducted a correlation analysis between CARM1 and checkpoint genes expression and found that CARM1 expression is highly correlated with CD276 in various cancer types (Fig. S5, see Additional file 9). Additionally, CARM1 expression has a certain correlation with the expression of multiple immune checkpoints in KICH, KIRC, KIRP, LGG, LIHC and THCA. In contrast, the expression of CARM1 is negatively correlated with most immune checkpoint molecules in SKCM and TGCT.

\section{CARM1-associated genes enrichment analysis}

In order to further study the biological significance of CARM1 gene in tumorigenesis, we screened CARM1 binding protein and expression related genes, and carried 


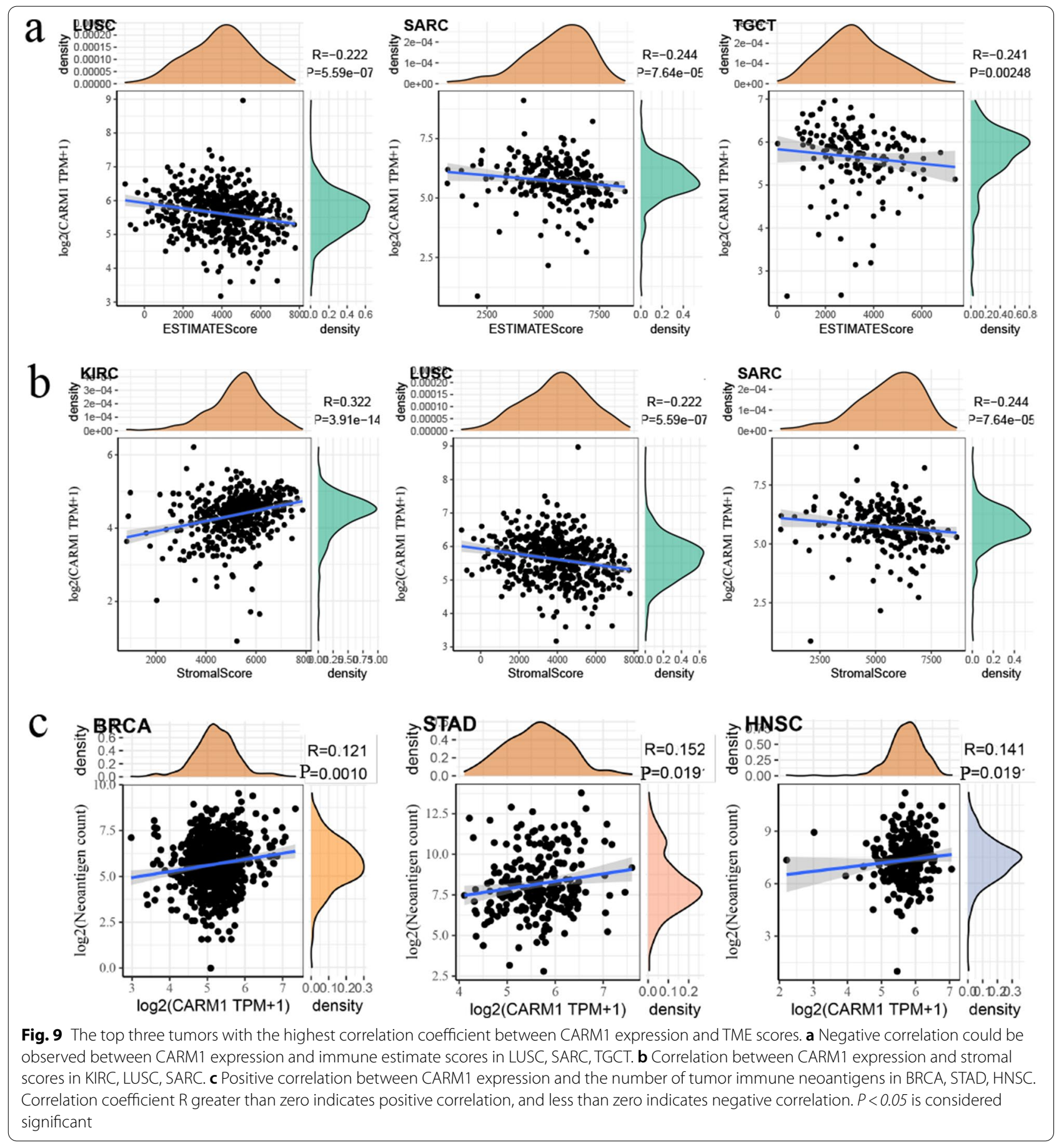

out a series of pathway enrichment analysis. STRING tool was applied to obtain the top 50 CARM1 binding proteins, which have been shown in the form of interaction network in Fig. 10a. Then, we used the GEPIA2 to acquire the first 100 genes related to CARM1 expression, and the top 6 genes with the highest correlation are shown in the form of scatter diagram in Fig. 10b.
Corresponding heatmap data also indicate that CARM1 is positively correlated with the above 6 genes in most cancer types (Fig. 10c). Combined with the above two databases, we conducted KEGG enrichment analysis. The results show that the effect of CARM1 on tumor pathogenesis may be related to transcriptional misregulation and viral carcinogenesis (Fig. 10d). 


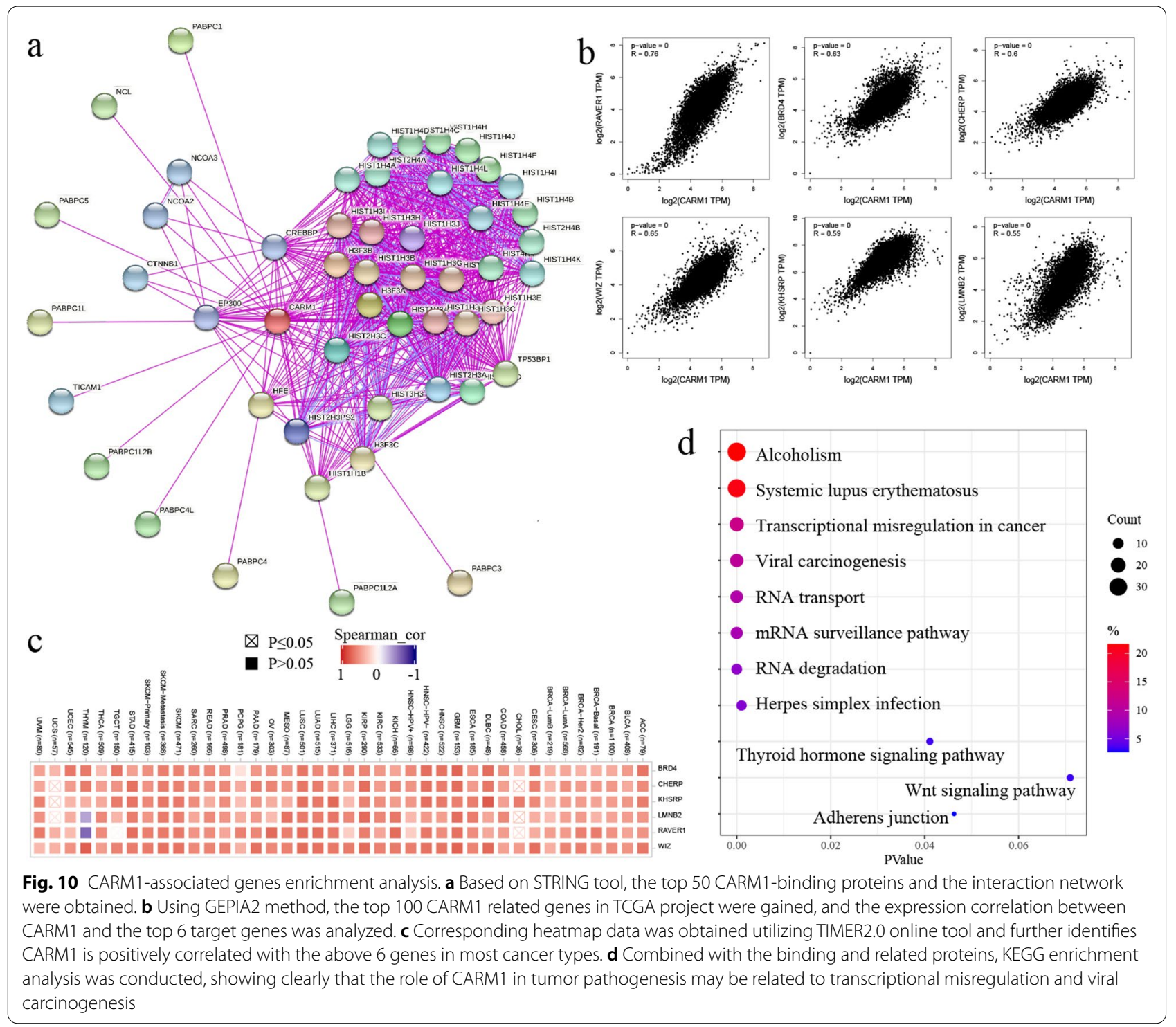

\section{Discussion}

CARM1 has been well known as a transcriptional coactivator, it has also been found to be crucial in regulation of metabolism, autophagy, RNA regulation and early mammalian development. In addition, increasing evidences indicate that CARM1 exerts an impact on the occurrence and development of tumors. After literature search, we found there is still a lack of research reports on pan-cancer analysis of CARM1. Therefore, based on the data from TCGA, CPTAC and other databases, we comprehensively detected the potential significance of CARM1 expression in various cancers from the perspectives of gene expression, gene alteration, immune microenvironment and related signaling pathways. Overexpression of CARM1 in most tumors was first verified, which is also associated with the pathological stage of some tumors, such as ACC, ESCA, KICH and UCS. Survival analysis results from GEPIA2 indicate that high expression of CARM1 is a significant adverse prognostic factor in ACC, BLCA, LGG, MESO, SKCM and other tumors. Overall, according to the analysis conducted by Kaplan Meier plotter, among the five tumors provided by the website, the low expression CARM1 group has a better clinical prognosis. However, subgroup analysis shows that the prognosis value of the CARM1 expression level vary in some subgroups. For example, the effect of CARM1 expression on prognosis in breast cancer is related to the state of HER2. In the HER2 ${ }^{-}$group, the prognosis of CARM1 low expression group is better 
than that of high expression group, while the prognosis of HER2 ${ }^{+}$subgroup is the opposite, which has been confirmed in previous studies [36]. In patients with hepatocellular carcinoma, high expression of CARM1 has a better prognosis in patients with hepatitis virus infection, while low expression of CARM1 is associated with favorable clinical prognosis of OS, FP and PPS, especially in high stage, specific TNM grading and pathological classification. Additionally, the analysis data obtained from cBioPortal tool shows that the mutation of CARM1 gene is related to the poor prognosis of colon cancer, which has been proved to be highly expressed CARM1 protein by CPTAC analysis tool. Therefore, on the premise of fully considering subgroup factors, CARM1 expression level is expected to be a good prognostic index. This study also analyzed a series of immune related factors of CARM1. Based on the results of immune cells infiltration, immune and matrix score as well as co-expression analysis of MMRs, methyltransferases genes and immune checkpoint genes, we found that CARM1 potentially affects the tumor immune microenvironment in most tumors, especially ACC, LUAD, LUSC, STAD, HNSC, THYM, etc. It is a direction worth exploring to clarify how CARM1 affects tumor immunity.

Our study conducts a comprehensive analysis of CARM1 in pan-cancer, which could provide clues for detecting its prognostic value and potential immunological function in tumor therapy. Information on various indicators suggesting the potential significance of CARM1 in different tumors has been summarized in Table 4, where the overall impact and conclusions about CARM1 on a certain tumor can be quickly found. Nevertheless, there are still some limitations in the present study. Although the correlation analysis between the gene expression of CARM1 and immune related factors implies the relevancy between them, it is not enough to capture detailed interaction. The concrete mechanisms of CARM1 affecting tumor immune microenvironment still needs further experimental verification.

\section{Conclusions}

In the light of big data analysis based on multiple databases, we revealed that the expression level and mutation degree of CARM1 are significantly related with clinical prognosis of patients with various tumors, indicating that CARM1 is expected to become an effective prognostic index. In the process of exploring mechanisms of CARM1 involved in tumor progression, we correlated probable causes from the perspective of several immune related elements and signaling pathways, which would be conducive to provide clues to support further molecular mechanism exploration, and may furnish an immune based antitumor strategy to provide a reference for more accurate and personalized immunotherapy in the future.

\section{Methods}

\section{Acquisition of gene information and protein structure}

The detailed genomic location information of CARM1 gene was obtained by querying UCSC website (http:// genome.ucsc.edu/). Then, the protein structure diagrams of CARM1 containing conserved regions in different species were gained and analyzed by using the Homologene module in NCBI website (https://www.ncbi.nlm.nih.gov/ homologene/). Additionally, the phylogenetic tree of CARM1 in different species was also acquired by using the COBALT online tool of NCBI (https://www.ncbi.nlm. nih.gov/tools/cobalt/).

\section{Gene expression analysis at mRNA level}

The expression data in different tissues, blood cells and tumor cell lines under physiological conditions were obtained by inputting "CARM1" in HPA database (https://www.proteinatlas.org/humanproteome/ pathology). Using the "Gene_DE" module of TIMER2 (tumor immune estimation resource, version 2) website (http://timer.cistrome.org/), the expression difference of CARM1 between tumors and corresponding normal tissues in TCGA project was observed. As for tumors without normal control, "Expression analysis-Box Plots" module of GEPIA (Gene Expression Profiling Interactive Analysis, version 2) was applied to acquire expression data from GTEx (Genotype-Tissue expression) database, under the settings of " $P$-value cutoff $=0.01, \log 2 \mathrm{FC}$ (fold change) cutoff $=1$ " and "Match TCGA normal and GTEx data" (http://gepia2.cancer-pku.cn/) [37]. We also utilize the "pathological staging map" module of GEPIA2 to obtain the violin plot of CARM1 expression in different pathological stages (stage I, II, III and IV) of all TCGA tumors.

\section{Gene expression analysis at protein level}

To evaluate the CARM1 expression difference from the protein expression level, immunohistochemistry images of tumor tissues and corresponding normal tissues were downloaded from HPA and analyzed. Further, we obtained CPTAC (clinical proteome tumor analysis alliance) dataset from the UALCAN portal (http://ualcan. path.uab.edu/analysis-prot.html) and conducted protein expression analysis of various tumors.

\section{Survival prognosis analysis}

Utilizing the "Survival Map" and "Survival Analysis" module of GEPIA 2, the OS (overall survival) and DFS 


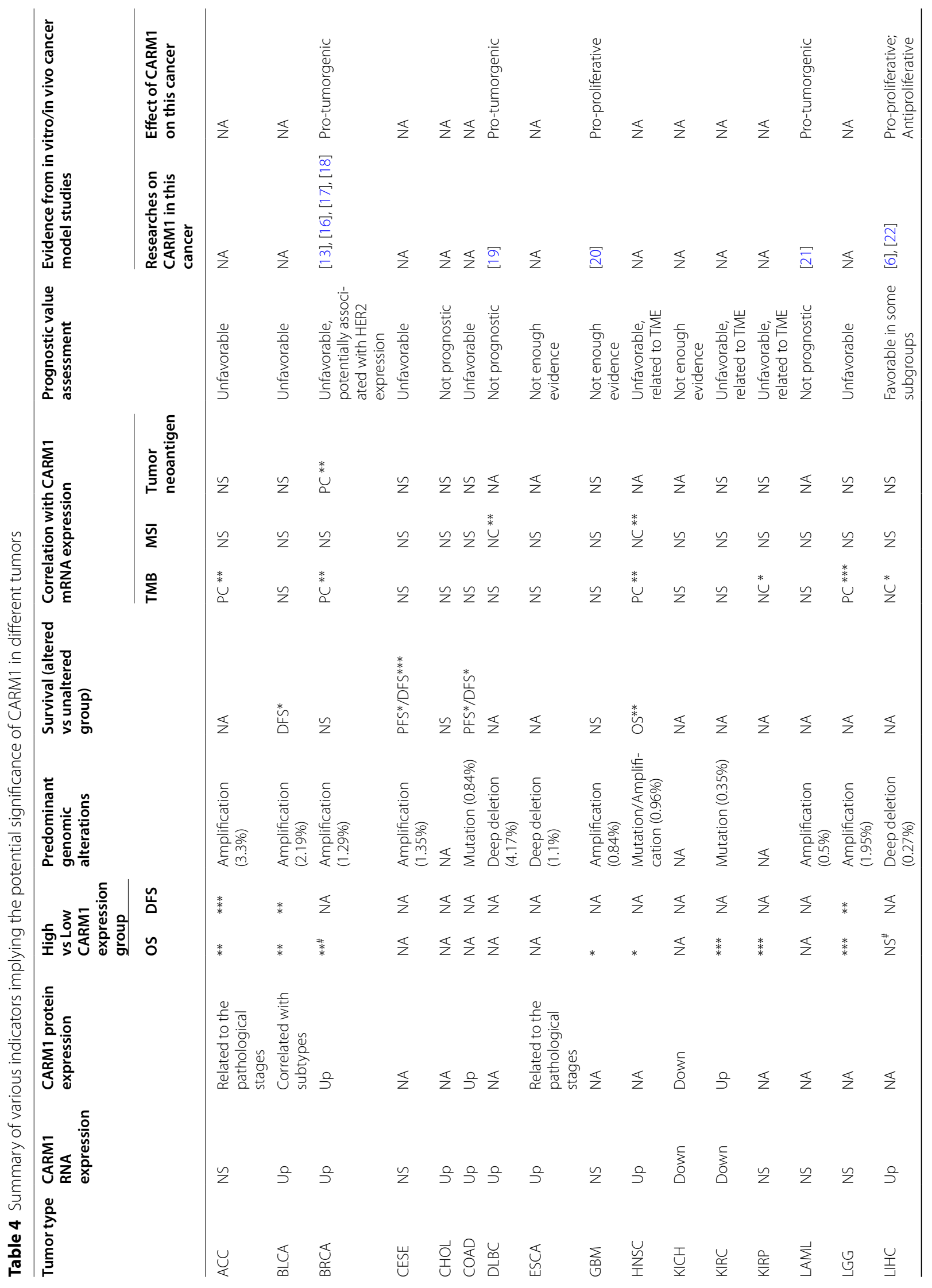




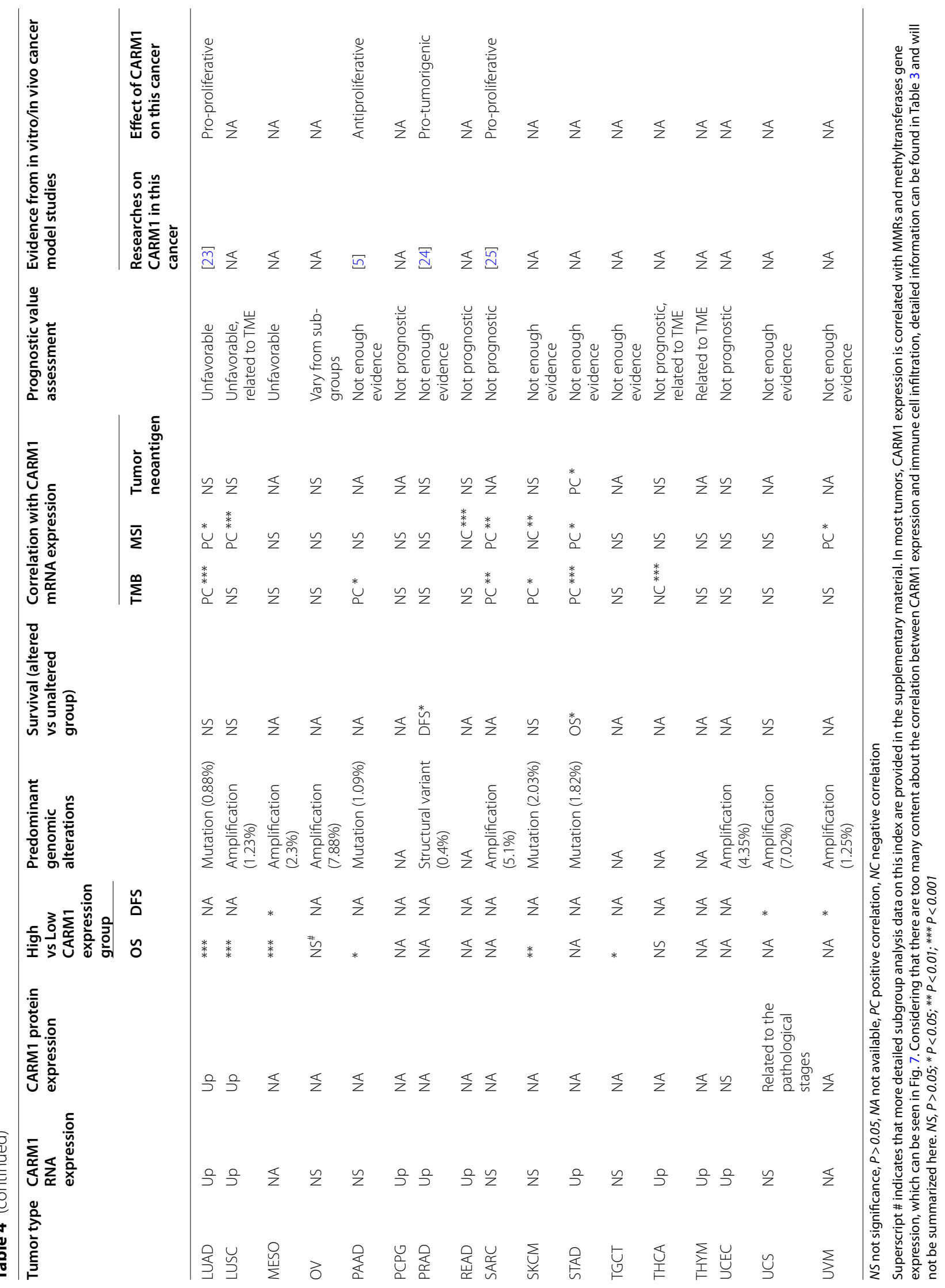


(disease-free survival) significance map data and survival map of CARM1 in all TCGA tumors can be obtained respectively. Cutoff-high (50\%) and cutoff-low (50\%) values were used as the expression thresholds to split the high-expression and low-expression cohorts. The association between CARM1 expression and survival in pan-cancer was also verified by Kaplan-Meier Plotter (https://kmplot.com/analysis/), which pools the different GEO datasets for a series of analyses of OS, DMFS (distant metastasis-free survival), RFS (relapse-free survival), PPS (post-progression survival), FP (first progression), DSS (disease-specific survival), and PFS (progress-free survival). The five types of tumor cases were split by setting "autoselect best cutoff" and the hazard ratio (HR), log-rank P-value and 95\% confidence intervals were computed, as well as the Kaplan-Meier survival plots were generated. We also set up other grouping factors to obtain subgroup analysis data of CARM1 mRNA expression and prognosis.

\section{Gene alteration and survival analysis}

Analysis of gene alteration of CARM1 in pan-cancer was conducted by querying the Cbioportal tool (http://www. cbioportal.org/) [38]. The "TCGA Pan Cancer Atlas Studies" module was selected to get the genetic alteration characteristics of CARM1. The "comparison" module was also used to obtain survival prognosis data of cancer cases from TCGA (with or without CARM1 gene alteration), and Kaplan Meier plots with log-rank $P$-value were generated.

\section{Tumor immune microenvironment analysis}

To explore the correlation between gene expression and immunotherapeutic response biomarkers TMB and MSI, sangerbox tool was used with the query of "CARM1" (http://sangerbox.com/Tool). TMB was calculated as the total mutation incidences per million base pair, and MSI was counted by the number of insertion or deletion events that occurred in repeating sequences of genes. Spearman method was used and the P-value as well as partial correlation value were obtained. We also conducted a co-expression analysis between CARM1 and mismatch repair genes (MMRs), methyltransferases as well as acknowledged immune checkpoints genes respectively. The images were modified using the software Adobe Illustrator.

To predict the presence of infiltration stromal or immune cells in pan-cancer tissues, R-package "estimate" and "limma" were used to calculate the scores of immune and stromal cells. As a database derived-web tool for immune cell infiltration calculation, TIMER provides the infiltration scores of many common types of immune cells [39]. Herein, we downloaded the infiltration data from it and used to test the correlation with CARM1 expression. The TIMER, EPIC, MCPCOUNTER, XCELL, CIBERSORT, CIBERSORT-ABS and QUANTISEQ algorithms were applied for immune cells infiltration calculation.

\section{CARM1-associated genes enrichment analysis}

On the STRING website (https://string-db.org/), we firstly input the gene name and the organism "Homo sapiens", then set the parameters as Network type [full network], meaning of network edges [evidence], active interaction sources [experiments], minimum required interaction score [medium confidence (0.400)] and max number of interactors to show [" "no more than 50 interactors" in 1st shell] and finally obtained the top 50 binding proteins network of CARM1. According to the data sets of all TCGA tumors and normal tissues, the "Similar Gene Detection" module of GEPIA2 was used to obtain the top 100 CARM1 related genes. The "Correlation Analysis" module of GEPIA2 was also used to perform a pairwise gene Pearson correlation analysis between CARM1 and the selected genes. The log2 TPM was applied for the dot plot and the P-value as well as the correlation coefficient (R) were indicated. After that, the "Gene_Corr" module of TIMER2 was applied to generate a heatmap containing the partial correlation Fcorand P-value in the purity-adjusted Spearman's rank correlation.

Combining the two sets of data, KEGG (Kyoto Encyclopedia of genes and genomes) pathway analysis was carried out by uploading the gene lists to DAVID (https://david.ncifcrf.gov/), Database for annotation, visualization, and integrated discovery). The analysis results are visualized with "ggplot2" $R$ software package.

\footnotetext{
Abbreviations

PRMT: Protein arginine methyltransferase; CARM1: Coactivator associated arginine methyltransferase 1; PH-like: Pleckstrin homology-like; SAM: S-adenosyl methionine; LSD1: Lysine demethylase 1; TCGA: The Cancer Genome Atlas; HPA: Human Protein Altas; TMB: Tumor mutation burden; MSI: Microsatellite instability; TIMER2: Tumor immune estimation resource, version 2; GEPIA: Gene Expression Profiling Interactive Analysis, version 2; GTEx: Genotype-Tissue expression; CPTAC: Clinical proteome tumor analysis alliance; OS: Overall survival; DFS: Disease-free survival; DMFS: Distant metastasis-free survival; RFS: Relapse-free survival; PPS: Post-progression survival; FP: First progression; DSS: Disease-specific survival; PFS: Progress-free survival; HR: Hazard ratio; MMRs: Mismatch repair genes; DAVID: Database for annotation, visualization, and integrated discovery; FANTOM5: Function annotation of the mammalian genome 5; BLCA: Bladder urothelial carcinoma; BRCA: Breast invasive carcinoma; CHOL: Cholangio carcinoma; COAD: Colon adenocarcinoma; ESCA: Esophageal carcinoma; HNSC: Head and neck squamous cell carcinoma; LIHC: Liver hepatocellular carcinoma; LUAD: Lung adenocarcinoma; LUSC: Lung squamous cell carcinoma; READ: Rectum adenocarcinoma; STAD: Stomach adenocarcinoma; THCA: Thyroid carcinoma; UCEC: Uterine corpus endometrial carcinoma; PCPG: Pheochromocytoma and paraganglioma; PRAD: Prostate adenocarcinoma; KICH: Kidney Chromophobe; KIRC: Kidney renal clear cell carcinoma; DLBC: Diffuse large B-cell lymphoma; THYM: Thymoma; IHC:
} 
Immunohistochemistry; ACC: Adrenocortical carcinoma; UCS: Uterine carcinosarcoma; KIRP: Kidney renal papillary cell carcinoma; LGG: Brain lower Grade Glioma; MESO: Mesothelioma; SKCM: Skin cutaneous melanoma; UVM: Uveal melanoma; ER: Eestrogen receptor; CESC: Cervical squamous cell carcinoma and endocervical adenocarcinoma; AML: Acute myeloid leukemia; TME: Tumor microenvironment; PAAD: Pancreatic adenocarcinoma; SARC: Sarcoma; TGCT: Testicular germ cell tumors.

\section{Supplementary Information}

The online version contains supplementary material available at https://doi. org/10.1186/s12863-021-01022-w.

Additional files 1: Figure S1. Structural characteristics and evolutionary relationship of CARM1 protein in different species.

Additional files 2: Figure S2. The RNA and protein expression profile of CARM1 in various cancers and blood cells.

Additional files 3: Figure S3. Tumors with no significant correlation between CARM1 expression and pathological stages.

Additional files 4: Table S1. Subgroup analysis on the correlation of CARM1 expression and prognosis of gastric cancer cases.

Additional files 5: Table S2. Subgroup analysis on the correlation of CARM1 expression and prognosis of liver cancer cases.

Additional files 6: Table S3. Subgroup analysis on the correlation of CARM1 expression and prognosis of ovarian cancer cases.

Additional files 7: Table S4. Subgroup analysis on the correlation of CARM1 expression and prognosis of lung cancer cases.

Additional files 8: Figure S4. Tumor prognosis information from Kaplan Meier plotter.

Additional files 9: Figure $\mathbf{S 5}$. Correlation between CARM1 and some known immune checkpoints mRNA expression in various cancers from TCGA.

\section{Acknowledgements}

We want to thank aforementioned databases for the availability of the data.

\section{Authors' contributions}

All authors have made substantial contributions to this review. Under the supervision of YHL, YQQ organized and drafted the work. HW, PYL, BYX, RH and YLY assisted to analyze the relevant data and revise the draft. All authors have read and approved the manuscript.

\section{Funding}

This work is supported by the Frontier Research Program of Guangzhou Regenerative Medicine and Health Guangdong Laboratory (2018GZR110105014), the Natural Science Foundation of Guangdong Province, China (2018B030311042).

\section{Availability of data and materials}

The authors certify that all the original data in this research could be obtained from a public database. The expression data of CARM1 are available in TIMER2 (http://timer.cistrome.org/), GEPIA2 (http://gepia2.cancer-pku.cn/) and UALCAN (http://ualcan.path.uab.edu/analysis-prot.html), which sources were from TCGA (https://portal.gdc.cancer.gov/) and CPTAC. Immunohistochemistry images were downloaded from HPA (https://www.proteinatlas.org/humanproteome/ pathology). The survival prognosis data could be found in GEPIA2 and verified by Kaplan-Meier Plotter (https://kmplot.com/analysis/). Gene alteration data of CARM1 can be found by querying the Cbioportal tool (http://www.cbioportal. org/). Immune related data were downloaded from TIMER2 and sangerbox (http:// sangerbox.com/Tool), which sources were from UCSC (https://xena.ucsc.edu/).

\section{Declarations}

Ethics approval and consent to participate Not applicable.

\section{Consent for publication}

Not applicable.

\section{Competing interests}

The authors declare that they have no competing interests.

\section{Author details}

'Department of Hematology, Zhujiang Hospital, Southern Medical University, No. 253 GongyeDadaoZhong, Guangzhou, Guangdong 510280, People's Republic of China. ${ }^{2}$ Bioland Laboratory (Guangzhou Regenerative Medicine and Health Guangdong Laboratory), Guangzhou, Guangdong 510005, People's Republic of China.

Received: 27 August 2021 Accepted: 23 December 2021

Published online: 16 January 2022

\section{References}

1. Chen D, Ma H, Hong H, Koh SS, Huang SM, Schurter BT, et al. Regulation of transcription by a protein methyltransferase. Science (New York, NY). 1999;284(5423):2174-7.

2. Shishkova E, Zeng H, Liu F, Kwiecien NW, Hebert AS, Coon JJ, et al. Global mapping of CARM1 substrates defines enzyme specificity and substrate recognition. Nat Commun. 2017;8:15571.

3. Troffer-Charlier N, Cura V, Hassenboehler P, Moras D, Cavarelli J. Functional insights from structures of coactivator-associated arginine methyltransferase 1 domains. EMBO J. 2007:26(20):4391-401.

4. Liu F, Ma F, Wang Y, Hao L, Zeng H, Jia C, et al. PKM2 methylation by CARM1 activates aerobic glycolysis to promote tumorigenesis. Nat Cell Biol. 2017;19(11):1358-70.

5. Wang YP, Zhou W, Wang J, Huang X, Zuo Y, Wang TS, et al. Arginine methylation of $\mathrm{MDH} 1$ by CARM1 inhibits glutamine metabolism and suppresses pancreatic cancer. Mol Cell. 2016;64(4):673-87.

6. Zhong $X Y$, Yuan $X M, X u$ YY, Yin M, Yan WW, Zou SW, et al. CARM1 methylates GAPDH to regulate glucose metabolism and is suppressed in liver cancer. Cell Rep. 2018;24(12):3207-23.

7. Shin HJ, Kim H, Oh S, Lee JG, Kee M, Ko HJ, et al. AMPK-SKP2-CARM1 signalling cascade in transcriptional regulation of autophagy. Nature. 2016;534(7608):553-7.

8. Wang L, Charoensuksai P, Watson NJ, Wang X, Zhao Z, Coriano CG, et al. CARM1 automethylation is controlled at the level of alternative splicing Nucleic Acids Res. 2013;41(14):6870-80.

9. Wang J, Wang L, Feng G, Wang Y, Li Y, Li X, et al. Asymmetric expression of LincGET biases cell fate in two-cell mouse embryos. Cell. 2018:175(7):1887-901.e18.

10. Karakashev S, Fukumoto T, Zhao B, Lin J, Wu S, Fatkhutdinov N, et al. EZH2 inhibition sensitizes CARM1-high, homologous recombination proficient ovarian cancers to PARP inhibition. Cancer Cell. 2020;37(2):157-67 e6.

11. LuW, Yang C, He H, Liu H. The CARM1-p300-c-Myc-max (CPCM) transcriptional complex regulates the expression of CUL $4 \mathrm{~A} / 4 \mathrm{~B}$ and affects the stability of CRL4 E3 ligases in colorectal cancer. Int J Biol Sci. 2020;16(6):1071-85.

12. Wu D, He J, Zhang W, Wang $K$, Jin $S$, Li J, et al. CARM1 promotes non-small cell lung cancer progression through upregulating CCNE2 expression. Aging (Albany NY). 2020;12(11):10578-93.

13. Peng BL, Li WJ, Ding JC, He YH, Ran T, Xie BL, et al. A hypermethylation strategy utilized by enhancer-bound CARM1 to promote estrogen receptor alpha-dependent transcriptional activation and breast carcinogenesis. Theranostics. 2020;10(8):3451-73.

14. Bennesch MA, Segala G, Wider D, Picard D. LSD1 engages a corepressor complex for the activation of the estrogen receptor alpha by estrogen and CAMP. Nucleic Acids Res. 2016:44(18):8655-70.

15. Mann M, Cortez V, Vadlamudi R. PELP1 oncogenic functions involve CARM1 regulation. Carcinogenesis. 2013;34(7):1468-75.

16. Liu J, Feng J, Li L, Lin L, Ji J, Lin C, et al. Arginine methylation-dependent LSD1 stability promotes invasion and metastasis of breast cancer. EMBO Rep. 2020;21(2):e48597.

17. Cai XC, Zhang T, Kim EJ, Jiang M, Wang K, Wang J, et al. A chemical probe of CARM1 alters epigenetic plasticity against breast cancer cell invasion. Elife. 2019;8:e47110 
18. Wang L, Zhao Z, Meyer MB, Saha S, Yu M, Guo A, et al. CARM1 methylates chromatin remodeling factor BAF155 to enhance tumor progression and metastasis. Cancer Cell. 2016;30(1):179-80.

19. Veazey KJ, Cheng D, Lin K, Villarreal OD, Gao G, Perez-Oquendo M, et al. CARM1 inhibition reduces histone acetyltransferase activity causing synthetic lethality in CREBBP/EP300-mutated lymphomas. Leukemia. 2020;34(12):3269-85.

20. Wang F, Zhang J, Ke X, Peng W, Zhao G, Peng S, et al. WDR5-Myc axis promotes the progression of glioblastoma and neuroblastoma by transcriptional activating CARM1. Biochem Biophys Res Commun. 2020;523(3):699-706.

21. Vu LP, Perna F, Wang L, Voza F, Figueroa ME, Tempst P, et al. PRMT4 blocks myeloid differentiation by assembling a methyl-RUNX1-dependent repressor complex. Cell Rep. 2013;5(6):1625-38.

22. Osada S, Suzuki S, Yoshimi C, Matsumoto M, Shirai T, Takahashi S, et al. Elevated expression of coactivator-associated arginine methyltransferase 1 is associated with early hepatocarcinogenesis. Oncol Rep. 2013;30(4):1669-74

23. Meng J, Chen X, Han Z. PFKFB4 promotes lung adenocarcinoma progression via phosphorylating and activating transcriptional coactivator SRC-2. BMC Pulm Med. 2021;21(1):60.

24. Hong H, Kao C, Jeng MH, Eble JN, Koch MO, Gardner TA, et al. Aberrant expression of CARM1, a transcriptional coactivator of androgen receptor, in the development of prostate carcinoma and androgen-independent status. Cancer. 2004;101(1):83-9.

25. Li S, Cheng D, Zhu B, Yang Q. The overexpression of CARM1 promotes human osteosarcoma cell proliferation through the pGSK3beta/betacatenin/cyclinD1 signaling pathway. Int J Biol Sci. 2017;13(8):976-84.

26. Ritterhouse LL. Tumor mutational burden. Cancer Cytopathol. 2019;127(12):735-6.

27. Yang G, Zheng RY, Jin ZS. Correlations between microsatellite instability and the biological behaviour of tumours. J Cancer Res Clin Oncol. 2019;145(12):2891-9.

28. Li SKH, Martin A. Mismatch repair and colon cancer: mechanisms and therapies explored. Trends Mol Med. 2016;22(4):274-89.

29. Moore LD, Le T, Fan G. DNA methylation and its basic function. Neuropsychopharmacology. 2013;38(1):23-38.

30. Kumar S, Zeng Z, Bagati A, Tay RE, Sanz LA, Hartono SR, et al. CARM1 inhibition enables immunotherapy of resistant tumors by dual action on tumor cells and T cells. Cancer Discov. 2021;11(8):2050-71.

31. Fridman WH, Galon J, Dieu-Nosjean MC, Cremer I, Fisson S, Damotte D, et al. Immune infiltration in human cancer: prognostic significance and disease control. Curr Top Microbiol Immunol. 2011;344:1-24.

32. Lin B, Du L, Li H, Zhu X, Cui L, Li X. Tumor-infiltrating lymphocytes: warriors fight against tumors powerfully. Biomed Pharmacother. 2020;132:110873.

33. Sahai E, Astsaturov I, Cukierman E, DeNardo DG, Egeblad M, Evans RM, et al. A framework for advancing our understanding of cancer-associated fibroblasts. Nat Rev Cancer. 2020;20(3):174-86.

34. Peng $M, M o Y, W a n g ~ Y, W u$, Z, Zhang $Y$, Xiong F, et al. Neoantigen vaccine: an emerging tumor immunotherapy. Mol Cancer. 2019;18(1):128.

35. He X, Xu C. Immune checkpoint signaling and cancer immunotherapy. Cell Res. 2020:30(8):660-9.

36. Davis MB, Liu X, Wang S, Reeves J, Khramtsov A, Huo D, et al. Expression and sub-cellular localization of an epigenetic regulator, co-activator arginine methyltransferase 1 (CARM1), is associated with specific breast cancer subtypes and ethnicity. Mol Cancer. 2013;12(1):40.

37. Tang Z, Kang B, Li C, Chen T, Zhang Z. GEPIAZ: an enhanced web server for large-scale expression profiling and interactive analysis. Nucleic Acids Res. 2019:47(W1):W556-W60.

38. Cerami E, Gao J, Dogrusoz U, Gross BE, Sumer SO, Aksoy BA, et al. The cBio cancer genomics portal: an open platform for exploring multidimensional cancer genomics data. Cancer Discov. 2012;2(5):401-4.

39. LiT, Fan J, Wang B, Traugh N, Chen Q, Liu JS, et al. TIMER: a web server for comprehensive analysis of tumor-infiltrating immune cells. Cancer Res. 2017;77(21):e108-e10.

\section{Publisher's Note}

Springer Nature remains neutral with regard to jurisdictional claims in published maps and institutional affiliations.

Ready to submit your research? Choose BMC and benefit from:

- fast, convenient online submission

- thorough peer review by experienced researchers in your field

- rapid publication on acceptance

- support for research data, including large and complex data types

- gold Open Access which fosters wider collaboration and increased citations

- maximum visibility for your research: over 100M website views per year

At BMC, research is always in progress.

Learn more biomedcentral.com/submissions 